\title{
Commutator of Marcinkiewicz Integral Operators on Herz-Morrey-Hardy Spaces with Variable Exponents
}

\author{
Omer Khalil ${ }^{1,2^{*}}$, Shuangping Tao ${ }^{1 *}$, Bechir Mahamat ${ }^{1}$ \\ ${ }^{1}$ College of Mathematics and Statistics, Northwest Normal University, Lanzhou, China \\ ${ }^{2}$ Faculty of Education, Sudan University of Science and Technology, Khartoum, Khartoum State, Sudan \\ Email: ^us.omer2008@sustech.edu, ^taosp@nwnu.edu.cn, bechirmahamatacyl@gmail.com
}

How to cite this paper: Khalil, O., Tao, S.P. and Mahamat, B. (2021) Commutator of Marcinkiewicz Integral Operators on Herz-Morrey-Hardy Spaces with Variable Exponents. Applied Mathematics, 12, 421448.

https://doi.org/10.4236/am.2021.125030

Received: April 6, 2021

Accepted: May 28, 2021

Published: May 31, 2021

Copyright $\odot 2021$ by author(s) and Scientific Research Publishing Inc. This work is licensed under the Creative Commons Attribution International License (CC BY 4.0).

http://creativecommons.org/licenses/by/4.0/

\begin{abstract}
In this paper, our aim is to prove the boundedness of commutators generated by the Marcinkiewicz integrals operator $\left[b, \mu_{\Omega}\right]$ and obtain the result with Lipschitz function and BMO function $f$ on the Herz-Morrey-Hardy spaces with variable exponents $H M K_{p(\cdot) \lambda}^{\alpha(\cdot), q}\left(\mathbb{R}^{n}\right)$.

\section{Keywords}

Marcinkiewicz Integral Operator, Herz-Morrey-Hardy Space, Commutator, Variable Exponent, Lipschitz Space
\end{abstract}

\section{Introduction}

Firstly in 1938, Marcinkiewicz [1] introduced the Marcinkiewicz integral. Next, the Marcinkiewicz integral operator has been studied extensively by many mathematicians in various fields. For example, Stain in [2] introduced the Marcinkiewicz integral operator related to the littlewood-Paley $g$ function on $\mathbb{R}^{n}$ and proved that $\mu_{\Omega}$ is of type $(p, p)$ for $1<p \leq 2$ and of week type $(1,1)$. In [3], Ding, Fan and Pan improved the above result and obtained the $L^{p}(1<p<\infty)$ and weighted $L^{p}(1<p<\infty)$ boundedness of the Marcinkiewicz cussed the boundedness for the commutator generated by the Marcinkiintegral $\mu$ under some weak conditions. Torchinsky and Wang in [4] discussed integral $\mu_{\Omega}$ and $B M O\left(\mathbb{R}^{n}\right)$ function on Lebesgue spaces $L^{p}\left(\mathbb{R}^{n}\right)$.

On the other hand, a class of functional spaces called Herz-Morrey-Hardy spaces with variable exponent has attracted great interest in recent years. We find that in successive studies in this field, in [5] [6] Xu, Yang introduced Herz- 
Morrey-Hardy spaces with variable exponents and their some applications. He obtained that certain singular integral operators are bounded from Herz-MorreyHardy spaces with variable exponents into Herz-Morrey spaces with variable exponents as an application of the atomic characterization. Also, he established their molecular decomposition, and by using their atomic and molecular decompositions, he gave the boundedness of a convolution type singular integral on Herz-Morrey-Hardy spaces with variable exponents. Omer in [7] proved the boundedness of commutators generated by the Calderón-Zygmund and used properties of variable exponent, $\mathrm{BMO}\left(\mathrm{R}^{\mathrm{n}}\right)$ function and Lipschitz function to prove this boundedness. Also, Yang in [8] established some boundedness for $T D^{\gamma}-D^{\gamma} T$ and $\left(T^{*}-T^{\#}\right) D^{\gamma}$ on the homogeneous Morrey-Herz-type Hardy spaces with variable exponents and studied Boundedness of Calderón-Zygmund operator on these spaces.

Suppose $\mathbb{S}^{n-1}(n \geq 2)$ denotes the unit sphere in $\mathbb{R}^{n}$ equipped with the normalized measure $\mathrm{d} \sigma$. Let $\Omega$ be homogenous function of degree zero and satisfies

$$
\int_{\mathbb{S}^{n-1}} \Omega\left(x^{\prime}\right) \mathrm{d} \sigma\left(x^{\prime}\right)=0,
$$

where $x^{\prime}=x /|x|$ for any $x \neq 0$.

Then the Marcinkiewicz integral operator $\mu_{\Omega}$ is defined by

$$
\mu_{\Omega}(f)(x)=\left(\int_{0}^{\infty}\left|F_{\Omega, t}(f)(x)\right|^{2} \frac{\mathrm{d} t}{t^{3}}\right)^{1 / 2},
$$

where

$$
F_{\Omega, t}(f)(x)=\int_{|x-y| \leq 1} \frac{\Omega(x-y)}{|x-y|^{n-1}} f(y) \mathrm{d} y .
$$

Let $b \in \operatorname{Lip}_{\gamma}\left(\mathbb{R}^{n}\right)$ and $b \in B M O$ be a locally integrable function on $\mathbb{R}^{n}$, the commutator generated by the Marcinkiewicz integral $\mu_{\Omega}$ and $b$ is defined by

$$
\left[b, \mu_{\Omega}\right]=\left(\int_{0}^{\infty}\left|\int_{|x-y| \leq t} \frac{\Omega(x-y)}{|x-y|^{n-1}}[b(x)-b(y)] f(y) \mathrm{d} y\right|^{2} \frac{\mathrm{d} t}{t^{3}}\right)^{1 / 2} .
$$

Motivated by [6] and [7], the aim of this paper is to study the boundedness for the commutator of Marcinkiewicz integral operator $\left[b, \mu_{\Omega}\right]$ on the Herz-MorreyHardy space with variable exponent where $\Omega \in L^{s}\left(S^{n-1}\right)$ for $s \geq 1$, with BMO function and Lipschitz function, we will define The definitions of the Morrey-Herz spaces with variable exponents, the Morrey-Herz-Hardy spaces with variable exponents (which will be defined in the next section), and the preliminary lemmas are presented in Section 2. In Section 3, we will prove the boundedness of the commutator of Marcikiewicz integrals on Herz-Morrey-Hrdy spaces with variable exponent with $b \in \operatorname{Lip}_{\gamma}\left(\mathbb{R}^{n}\right)$. Lastly, in Section 4 we will prove the boundedness of the commutator of Marcikiewicz integrals on HerzMorrey-Hrdy spaces with variable exponent with function $b \in B M O\left(\mathbb{R}^{n}\right)$. 
A given open set $\Omega \subset \mathbb{R}^{n}$ and a measurable function $p(\cdot): \Omega \rightarrow[1, \infty)$, $L^{p(\cdot)}(\Omega)$ denotes the set of measurable function $f$ on $\Omega$ such that for some $\lambda>0$,

$$
L^{p(\cdot)}(\Omega)=\left\{f \text { is measurable }: \int_{\Omega}\left(\frac{|f(x)|}{\eta}\right)^{p(x)} \mathrm{d} x<\infty \text { for some constant } \eta>0\right\},
$$

the space $L_{L o c}^{p(\cdot)}(\Omega)$ is defined by

$$
L_{\text {Loc }}^{p(\cdot)}(\Omega)=\left\{f \text { is measurable }: f \in L^{p(\cdot)}(K) \text { for all compact } K \subset \Omega\right\} .
$$

The Lebesgue spaces $L^{p(\cdot)}(\Omega)$ is Banach spaces with the norm defined by

$$
\|f\|_{L^{p()}(\Omega)}=\inf \left\{\eta>0: \int_{\Omega}\left(\frac{|f(x)|}{\eta}\right)^{p(x)} \mathrm{d} x \leq 1\right\}
$$

where $p_{-}=\operatorname{essinf}\{p(x): x \in \Omega\}>1, \quad p_{+}=\operatorname{esssup}\{p(x): x \in \Omega\}<\infty$.

Denotes $p^{\prime}(x)=p(x) /(p(x)-1)$. Let $M$ be the Hardy-Littlewood maximal operator. We denote $\mathcal{B}(\Omega)$ to be the set of all functions $p(\cdot) \in \mathcal{P}(\Omega)$ satisfying the $M$ is bounded on $L^{p(\cdot)}(\Omega)$.

Definition 1.1. [6]

Let $0<q \leq \infty, p(\cdot) \in \mathcal{P}\left(\mathbb{R}^{n}\right), \quad 0 \leq \lambda<\infty$. Let $\alpha(\cdot)$ be a bounded real-valued measurable function on $\mathbb{R}^{n}$. The nonhomogeneous Morrey-Herz space $M K_{p(\cdot), \lambda}^{\alpha(\cdot),}\left(\mathbb{R}^{n}\right)$ and homogeneous Morrey-Herz space with variable exponents $M \dot{K}_{p(\cdot), \lambda}^{\alpha(\cdot),}\left(\mathbb{R}^{n}\right)$ are respectively defined by

$$
M K_{p(\cdot), \lambda}^{\alpha(\cdot), q}:=\left\{f \in L_{\text {loc }}^{p(\cdot)}\left(\mathbb{R}^{n} \backslash\{0\}\right):\|f\|_{\left.M K_{p(\cdot), \lambda}^{\alpha(\cdot),}<\infty\right\},}\right.
$$

and

$$
M \dot{K}_{p(\cdot), \lambda}^{\alpha(\cdot), q}:=\left\{f \in L_{L o c}^{p(\cdot)}\left(\mathbb{R}^{n} \backslash\{0\}\right):\|f\|_{M \dot{K}_{p(\cdot), \lambda}^{\alpha(\cdot), q}}<\infty\right\},
$$

where

$$
\begin{gathered}
\|f\|_{M K_{(P(), \lambda}^{\alpha(\cdot), q}}:=\sup _{L \in \mathbb{N}_{0}} 2^{-L \lambda}\left(\sum_{k=0}^{L}\left\|2^{k \alpha(\cdot)} f \tilde{\chi}_{k}\right\|_{L^{p(\cdot)}}^{q}\right)^{1 / q}, \\
\|f\|_{M \dot{K}_{p(\cdot), \lambda}^{\alpha(\cdot),}}:=\sup _{L \in \mathbb{Z}} 2^{-L \lambda}\left(\sum_{k=-\infty}^{L}\left\|2^{k \alpha(\cdot)} f \chi_{k}\right\|_{L^{p(\cdot)}}^{q}\right)^{1 / q} .
\end{gathered}
$$

Definition 1.2. [9]

For all $0<\gamma \leq 1$, the Lipschitz space $\operatorname{Lip}_{\gamma}\left(\mathbb{R}^{n}\right)$ is defined by

$$
\operatorname{Lip}_{\gamma}=\left\{f:\|f\|_{L i p_{\gamma}}=\sup _{x, y \in \mathbb{R}^{n} ; x \neq y} \frac{|f(x)-f(y)|}{|x-y|^{\gamma}}<\infty\right\} .
$$

Definition 1.3. [5]

Let $\alpha(\cdot) \in L^{\infty}\left(\mathbb{R}^{n}\right), p(\cdot) \in \mathcal{P}\left(\mathbb{R}^{n}\right), 0<q \leq \infty, 0 \leq \lambda<\infty$ and $N>n+1$. The 
nonhomogeneous Herz-Morrey-Hardy space with variable exponent $H M K_{p(\cdot) \lambda}^{\alpha(\cdot), q}\left(\mathbb{R}^{n}\right)$ and homogeneous Herz-Morrey-Hardy space with variable exponents $H M \dot{K}_{p(\cdot) \lambda}^{\alpha(\cdot), q}\left(\mathbb{R}^{n}\right)$ are respectively defined by

$$
\begin{aligned}
& H \operatorname{HK}_{p(\cdot) \lambda}^{\alpha(\cdot), q}\left(\mathbb{R}^{n}\right):=\left\{f \in \mathcal{S}^{\prime}\left(\mathbb{R}^{n}\right):\|f\|_{H M K_{p(\cdot), \lambda}^{\alpha(\cdot) q}}:=\left\|G_{N} f\right\|_{M K_{p(\cdot) \lambda}^{\alpha(\cdot), q}}<\infty\right\}, \\
& H \dot{K}_{p(\cdot) \lambda}^{\alpha(\cdot), q}\left(\mathbb{R}^{n}\right):=\left\{f \in \mathcal{S}^{\prime}\left(\mathbb{R}^{n}\right):\|f\|_{H M \dot{K}_{p(\cdot), \lambda}^{\alpha(\cdot), q}}:=\left\|G_{N} f\right\|_{M \dot{K}_{p(\cdot) \lambda}^{\alpha(\cdot), q}}<\infty\right\} .
\end{aligned}
$$

Definition 1.4. [10] (Hölder's inequality) Let $\alpha>1$ and $1 / \alpha+1 / \beta=1$. Then the discrete and integral forms of Hölder's inequality are given as

$$
\int_{a}^{b}|f(x) g(x)| \mathrm{d} x \leq\left(\int_{a}^{b}|f(x)|^{\alpha}\right)^{1 / \alpha}\left(\int_{a}^{b}|g(x)|^{\beta}\right)^{1 / \beta},
$$

for continuous function $f$ and $g$ on $[a, b]$.

Definition 1.5. [10] (Minkowski's inequality) Let $u>1$. Then the discrete and integral forms of Minkowski's inequality are given as

$$
\left(\int_{a}^{b}|f(x)+g(x)|^{u} \mathrm{~d} x\right)^{1 / u} \leq\left(\int_{a}^{b}|f(x)|^{u}\right)^{1 / u}+\left(\int_{a}^{b}|g(x)|^{u}\right)^{1 / u},
$$

for continuous function $f$ and $g$ on $[a, b]$. for more general functions can be obtained naturally. A further generalization is: If $u>1$, then

$$
\left(\int\left(\int|f(x, y)| \mathrm{d} y\right)^{u} \mathrm{~d} x\right)^{1 / u} \leq \int\left(\int|f(x)|^{u} \mathrm{~d} x\right)^{1 / u} \mathrm{~d} y .
$$

\section{Preliminaries}

In this section, we give some preliminaries which we used to prove theorems.

Lemma 2.1. [11] Let $p(\cdot) \in \mathcal{P}\left(\mathbb{R}^{n}\right)$. Then for any $f \in L^{p(\cdot)}$ and $g \in L^{p^{\prime}(\cdot)}$, we have

$$
\int_{\mathbb{R}^{n}}|f(x) g(x)| \mathrm{d} x \leq C_{p}\|f\|_{L^{p(\cdot)}\left(\mathbb{R}^{n}\right)}\|g\|_{L^{p^{\prime}(\cdot)}\left(\mathbb{R}^{n}\right)},
$$

where $C_{p}=1+\frac{1}{p_{-}}-\frac{1}{p_{+}}$.

This inequality is called the generalized Hölder inequality with respect to the variable $L^{p(\cdot)}$ spaces.

Lemma 2.2. [12] Given $p(\cdot), p_{1}(\cdot), p_{2}(\cdot) \in \mathcal{P}\left(\mathbb{R}^{n}\right)$, for any

$$
\begin{aligned}
& f \in L^{p_{1}(\cdot)}\left(\mathbb{R}^{n}\right), g \in L^{p_{2}(\cdot)}\left(\mathbb{R}^{n}\right) \text {, when } \frac{1}{p(\cdot)}=\frac{1}{p_{2}(\cdot)}+\frac{1}{p_{1}(\cdot)} \text {, we get } \\
& \qquad\|f(x) g(x)\|_{L^{p(\cdot)}\left(\mathbb{R}^{n}\right)} \leq C\|f\|_{L^{p_{1}(\cdot)}\left(\mathbb{R}^{n}\right)}\|g\|_{L^{p_{2}}\left(\mathbb{R}^{n}\right)}, \\
& \text { where } C_{p_{1}, p_{2}}=\left[1+\frac{1}{p_{1-}}-\frac{1}{p_{1+}}\right]^{\frac{1}{p_{-}}} .
\end{aligned}
$$

Proposition 2.3. [13] If $q(\cdot) \in \mathcal{P}\left(\mathbb{R}^{n}\right)$ satisfies

$$
|q(x)-q(y)| \leq \frac{-C}{\log (|x-y|)}, \quad|x-y| \leq 1 / 2,
$$




$$
|q(x)-q(y)| \leq \frac{C}{\log (e+|x|)}, \quad|y| \geq|x|,
$$

then $q(\cdot) \in \mathfrak{B}\left(\mathbb{R}^{n}\right)$.

Lemma 2.4. [14] Let $k$ be a positive integer and $B$ be a ball in $\mathbb{R}^{n}$. Then we have that for all $b \in B M O\left(\mathbb{R}^{n}\right)$ and $i, j \in \mathbb{Z}$ with $i<j$, we have

1) $C^{-1}\|b\|_{*}^{k} \leq \sup _{B} \frac{1}{\left\|\chi_{B}\right\|_{L^{p()}\left(\mathbb{R}^{n}\right)}}\left\|\left(b-b_{B}\right) \chi_{B}\right\|_{L^{p(\cdot)}\left(\mathbb{R}^{n}\right)} \leq C\|b\|_{*}^{k}$,

2) $\left\|\left(b-b_{B_{i}}\right)^{k} \chi_{B_{j}}\right\|_{L^{(\cdot)}\left(\mathbb{R}^{n}\right)} \leq C(j-i)^{K}\|b\|_{*}^{k}\left\|\chi_{B_{j}}\right\|_{L^{q(\cdot)}\left(\mathbb{R}^{n}\right)^{n}}$,

where $B_{i}=\left\{x \in \mathbb{R}^{n}:|x| \leq 2^{i}\right\}$ and $B_{j}=\left\{x \in \mathbb{R}^{n}:|x| \leq 2^{j}\right\}$.

Lemma 2.5. [15] Let $q(\cdot) \in \mathfrak{B}\left(\mathbb{R}^{n}\right)$, then there exist positive constants $C>0$, such that for all balls $B \subset \mathbb{R}^{n}$ and all measurable subset $R \subset B$,

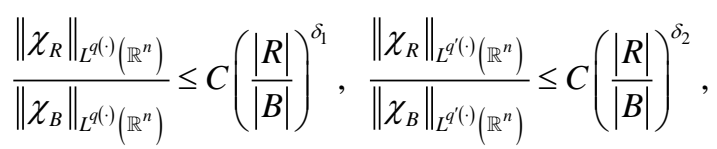

where $\delta_{1}, \delta_{2}$ are constants with $0<\delta_{1}, \delta_{2}<1$.

Lemma 2.6. [16] If $q(\cdot) \in \mathfrak{B}\left(\mathbb{R}^{n}\right)$, then there exists a constant $C>0$ such that for any balls $B$ in $\mathbb{R}^{n}$,

$$
\frac{1}{|B|}\left\|\chi_{B}\right\|_{L^{q(\cdot)}\left(\mathbb{R}^{n}\right)}\left\|\chi_{B}\right\|_{L^{q}(\cdot)\left(\mathbb{R}^{n}\right)} \leq C
$$

Lemma 2.7. [6] Let $0<q<\infty, p(\cdot) \in \mathfrak{B}\left(\mathbb{R}^{n}\right), 0<\lambda<\infty$, and $\alpha(\cdot) \in L^{\infty}\left(\mathbb{R}^{n}\right)$ be log-Hölder continuous both at the origin and infinity, $2 \lambda \leq \alpha(\cdot), n \delta_{2} \leq \alpha(0), \alpha_{\infty}<\infty, \quad \delta_{2}$ as in lemma 2.4. Then $f \in H M \dot{K}_{p(\cdot) \lambda}^{\alpha(\cdot), q}\left(\mathbb{R}^{n}\right)$ (or $H M K_{p(\cdot) \lambda}^{\alpha(\cdot), q}\left(\mathbb{R}^{n}\right)$ ) if and only if $f=\sum_{k=-\infty}^{\infty} \lambda_{k} f_{k}$ (or $f=\sum_{k=0}^{\infty} \lambda_{k} f_{k}$ ), in the sense of $f \in \mathcal{S}^{\prime}(\mathbb{R})^{n}$, where each $a_{k}$ is a central $(\alpha(\cdot), p(\cdot))$ atom with support contained in $B_{k}$ and

$$
\sup _{L \leq 0, L \in Z} 2^{-L \lambda} \sum_{k=-\infty}^{L}\left|\lambda_{k}\right|^{q}<\infty \quad \text { or }\left(\sup _{L \leq 0, L \in Z} 2^{-L \lambda} \sum_{k=0}^{L}\left|\lambda_{k}\right|^{q}\right),
$$

moreover

$$
\|f\|_{H M K_{p(\cdot), \alpha}^{\alpha(\cdot), q}} \approx \inf \sup _{L \leq 0, L \in Z} 2^{-L \lambda}\left(\sum_{k=-\infty}^{L}\left|\lambda_{k}\right|^{q}\right)^{1 / q}
$$

or

$$
\|f\|_{H M K_{p(\cdot), q}^{\alpha(\cdot), q}} \approx \inf \sup _{L \leq 0, L \in Z} 2^{-L \lambda}\left(\sum_{k=0}^{L}\left|\lambda_{k}\right|^{q}\right)^{1 / q},
$$

where infimum is taken over all above decomposition of $f$.

Lemma 2.8. [17] Let $q(\cdot) \in \mathcal{P}\left(\mathbb{R}^{n}\right), q \in(0, \infty]$ and $\lambda \in[0, \infty)$. If $\alpha(\cdot) \in L^{\infty}\left(\mathbb{R}^{n}\right) \cap \mathfrak{P}_{0}^{\log }\left(\mathbb{R}^{n}\right) \cap \mathfrak{P}_{\infty}^{\log }\left(\mathbb{R}^{n}\right)$, then

$$
\begin{aligned}
\|f\|_{M \dot{K}_{p(\cdot), \lambda}^{\alpha(\cdot), q}\left(\mathbb{R}^{n}\right)}^{q}= & \max \left\{\sup _{L \leq 0, L \in \mathbb{Z}} 2^{-L \lambda q} \sum_{k=-\infty}^{L} 2^{k q \alpha(0)}\left\|f \chi_{k}\right\|_{L^{p(\cdot)}}^{q},\right. \\
& \left.\sup _{L \leq 0, L \in Z} 2^{-L \lambda q}\left(\sum_{k=-\infty}^{-1} 2^{k q \alpha(0)}\left\|f \chi_{k}\right\|_{L^{p(\cdot)}}^{q}+\sum_{k=0}^{L} 2^{k q \alpha(\infty)}\left\|f \chi_{k}\right\|_{L^{p(\cdot)}}^{q}\right)\right\} .
\end{aligned}
$$


Lemma 2.9. [18] Let $\Omega$ satisfies $L^{r}$-Dini condition with $r \in[1, \infty)$. If there exist constants $C>0$ and $R>0$ such that $|y|<R / 2$, then for every $x \in \mathbb{R}^{n}$, we have

$$
\left(\int_{R<|R|<2 R}\left|\frac{\Omega(x-y)}{|x-y|}-\frac{\Omega(x)}{|x|}\right|^{r} \mathrm{~d} x\right)^{1 / r} \leq C R^{\left(\frac{n}{r}-n\right)}\left\{\frac{|y|}{R}+\int_{|y| / 2 R<\delta<|y| / R} \frac{\omega_{r}(\delta)}{\delta} \mathrm{d} \delta\right\} .
$$

Lemma 2.10. [15] Given $E$, let $q(\cdot) \in \mathcal{P}(E), f: E \times E \rightarrow \mathbb{R}^{n}$ be a measurable function (with respect to product measure) such that for almost every $y \in E, f(., y) \in L^{q(\cdot)}(E)$. Then

$$
\left\|\int_{E} f(., y) d y\right\|_{L^{q(\cdot)}(E)} \leq C \int_{E}\|f(., y)\|_{L^{(\cdot)}(E)} \mathrm{d} y .
$$

Lemma 2.11. [19] If $a>0,1 \leq s \leq \infty, 0 \leq d \leq s$ and $-n+(n-1) d / s<v<\infty$, then

$$
\left(\int_{|y| \leq a|x|}|y|^{v}|\Omega(x-y)|^{d} \mathrm{~d} y\right)^{1 / d} \leq C|x|^{(v+n) / d}\|\Omega\|_{L^{s}\left(s^{n-1}\right)} \cdot
$$

Lemma 2.12. [19] Let $q(\cdot) \in \mathcal{P}$ satisfies Proposition 2.3. Then

$$
\left\|\chi_{Q}\right\|_{L^{q(\cdot)}\left(\mathbb{R}^{n}\right)} \approx \begin{cases}|Q|^{\frac{1}{q(x)}} & \text { if }|Q| \leq 2^{n} \text { and } x \in Q \\ |Q|^{\frac{1}{q(\infty)}} & \text { if }|Q| \geq 1\end{cases}
$$

for every cube (or ball) $Q \in \mathbb{R}^{n}$, where $p(\infty)=\lim _{x \rightarrow \infty} p(x)$.

\section{Lipschitz Boundedness for the Commutator of Marcikiewicz Integrals Operator}

In this section, we prove the boundedness of the commutator of Marcikiewicz integrals on Herz-Morrey-Hrdy spaces with variable exponent so when $b \in \operatorname{Lip}_{\gamma}\left(\mathbb{R}^{n}\right)$ under some conditions.

Theorem 3.1.

Suppose that $b \in \operatorname{Lip}_{\gamma}\left(\mathbb{R}^{n}\right)$ with $0<\gamma \leq 1$. If $q_{1}(\cdot) \in \mathcal{P}\left(\mathbb{R}^{n}\right)$ satisfies proposition 2.3 with $q_{1}^{+}<n / \gamma, 1 / q_{1}(x)-1 / q_{2}(x)=\gamma / n, \Omega \in L^{s}\left(S^{n-1}\right)\left(s>q_{2}^{+}\right)$with $1 \leq s^{\prime}<q_{1}^{-}$and satisfies

$$
\int_{0}^{1} \frac{\Omega_{s}(\delta)}{\delta^{1+\gamma}} \mathrm{d} \delta<\infty
$$

let $0<p_{1} \leq q_{2}<\infty$ and $n \delta_{2} \leq \alpha<n \delta_{2}+\gamma$ or $\left(0<\max \left(n \delta_{2}, \alpha_{2}\right) \leq \alpha_{1}<n \delta_{2}+\gamma\right)$. Then the commutator $\left[b, \mu_{\Omega}\right]$ is bounded from $\operatorname{HMK}_{p(\cdot) \lambda}^{\alpha(\cdot), q}\left(\mathbb{R}^{n}\right)$ (or $\left.H M K_{p(\cdot) \lambda}^{\alpha(\cdot), q}\left(\mathbb{R}^{n}\right)\right)$ to $M \dot{K}_{p(\cdot) \lambda}^{\alpha(\cdot), q}\left(\mathbb{R}^{n}\right)$ (or $\left.M K_{p(\cdot) \lambda}^{\alpha(\cdot), q}\left(\mathbb{R}^{n}\right)\right)$.

To the proof the above theorem, we will recall the following lemma.

Lemma 3.1. [15]

Suppose that $b \in \operatorname{Lip}_{\gamma}\left(\mathbb{R}^{n}\right)$ with $0<\gamma \leq 1$. If $q_{1}(\cdot) \in \mathcal{P}\left(\mathbb{R}^{n}\right)$ satisfies Proposition 2.3 with $q_{1}^{+}<n / \gamma, 1 / q_{1}(x)-1 / q_{2}(x)=\gamma / n$ with $\Omega \in L^{s}\left(S^{n-1}\right)\left(s>q_{2}^{+}\right)$. Then the commutator $\left[b, \mu_{\Omega}\right]$ is bounded from $L^{q_{1}(\cdot)}\left(\mathbb{R}^{n}\right)$ to $L^{q_{1}(\cdot)}\left(\mathbb{R}^{n}\right)$. 
Next, we will give the Lipschitz estimate about the commutator $\left[b, \mu_{\Omega}\right]$ on Herz-Morrey-Hardy spaces with variable exponent.

\section{Proof Theorem 3.1:}

To prove this theorem, we only prove the homogeneous case. Let $f \in H M K_{p(\cdot) \lambda}^{\alpha(\cdot), q}\left(\mathbb{R}^{n}\right)$. By lemma 2.6 we have $f=\sum_{j=-\infty}^{\infty} \lambda_{j} f_{j}$ converged in $\mathcal{S}^{\prime}(\mathbb{R})^{n}$, where each $b_{j}$ is a central $(\alpha(\cdot), p(\cdot))$ atom with support contained in $B_{j}$ and

$$
\|f\|_{\text {нMK } K_{p(\cdot),}^{\alpha(\cdot), q}} \approx \inf \sup _{L \leq 0, L \in Z} 2^{-L \lambda}\left(\sum_{j=-\infty}^{L}\left|\lambda_{j}\right|^{q}\right)^{1 / q} .
$$

Here we denote $\Delta=\sup _{L \leq 0, L \in Z} 2^{-L \lambda} \sum_{k=-\infty}^{L}\left|\lambda_{k}\right|^{q}$. By lemma 2.8 we have

$$
\begin{gathered}
\left\|\left[b, \mu_{\Omega}\right](f)\right\|_{M K_{p(\cdot), \lambda}^{\alpha(\cdot), q}\left(\mathbb{R}^{n}\right)}^{q \leq 0, L \in Z}=\max \left\{\sup _{L \leq 0, L \in \mathbb{Z}} 2^{-L \lambda q} \sum_{k=-\infty}^{L} 2^{k q \alpha(0)}\left\|\left[b, \mu_{\Omega}\right](f) \chi_{k}\right\|_{L^{p(\cdot)}}^{q},\right. \\
\sup _{L \leq 0, L \in Z} 2^{-L \lambda q}\left(\sum_{k=-\infty}^{-1} 2^{k q \alpha(0)}\left\|\left[b, \mu_{\Omega}\right](f) \chi_{k}\right\|_{L^{p(\cdot)}}^{q}\right. \\
\left.\left.\quad+\sum_{k=0}^{L} 2^{k q \alpha(\infty)}\left\|\left[b, \mu_{\Omega}\right](f) \chi_{k}\right\|_{L^{p^{(\cdot)}}}^{q}\right)\right\} . \\
I=\sup _{L \leq 0, L \in \mathbb{Z}} 2^{-L \lambda q} \sum_{k=-\infty}^{L} 2^{k q \alpha(0)}\left\|\left[b, \mu_{\Omega}\right](f) \chi_{k}\right\|_{L^{p(\cdot)}}^{q}, \\
I I=\sum_{k=-\infty}^{-1} 2^{k q \alpha(0)}\left\|\left[b, \mu_{\Omega}\right](f) \chi_{k}\right\|_{L^{p(\cdot)}}^{q}, \\
I I I=\sup _{L \leq 0, L \in \mathbb{Z}} 2^{-L \lambda q} \sum_{k=0}^{L} 2^{k q \alpha(\infty)}\left\|\left[b, \mu_{\Omega}\right](f) \chi_{k}\right\|_{L^{p())}}^{q} .
\end{gathered}
$$

In beginning, we examine a function which we will use in proving

$$
\begin{aligned}
\left|\left[b, \mu_{\Omega}\right]\left(b_{j}\right)(x)\right| \leq & \left\{\int_{0}^{|x|}\left|\int_{|x-y| \leq t} \frac{|\Omega(x-y)|}{|x-y|^{n-1}}[b(x)-b(y)] b_{j}(y) \mathrm{d} y\right|^{2} \frac{\mathrm{d} t}{t^{3}}\right\}^{1 / 2} \\
& +\left\{\int_{|x|}^{\infty}\left|\int_{|x-y| \leq t} \frac{|\Omega(x-p y)|}{|x-y|^{n-1}}[b(x)-b(y)] b_{j}(y) \mathrm{d} y\right|^{2} \frac{\mathrm{d} t}{t^{3}}\right\}^{1 / 2} \\
& \simeq \Upsilon_{1}+\Upsilon_{2} .
\end{aligned}
$$

When $x \in A_{k}$ and $|x-y| \leq t$ with $t \leq|x|$, it follows from $j \leq k-2$ that $|x-y| \sim|x|$. We have

$$
\left|\frac{1}{|x-y|^{2}}-\frac{1}{|x|^{2}}\right| \leq \frac{|y|}{|x-y|^{3}} .
$$

Then by (3.1), the Minkowski's inequality, the generalized Hölder's inequality and the vanishing of the moment of $b_{j}$ we have

$$
\begin{aligned}
\Upsilon_{1} & \leq C \int_{\mathbb{R}^{n}}\left|\frac{|\Omega(x-y)|}{|x-y|^{2}}-\frac{|\Omega(x)|}{|x|^{2}}\right| b(x)-b(y)|| b_{j}(y) \mid\left(\int_{|x-y|}^{|x|} \frac{\mathrm{d} t}{t^{3}}\right)^{1 / 2} \mathrm{~d} y \\
& \leq C \int_{\mathbb{R}^{n}}\left|\frac{|\Omega(x-y)|}{|x-y|^{2}}-\frac{|\Omega(x)|}{|x|^{2}}\right| b(x)-b(y)|| b_{j}(y)|| \frac{1}{|x-y|^{2}}-\left.\frac{1}{|x|^{2}}\right|^{1 / 2} \mathrm{~d} y
\end{aligned}
$$




$$
\begin{aligned}
& \leq C \int_{\mathbb{R}^{n}}\left|\frac{|\Omega(x-y)|}{|x-y|^{2}}-\frac{|\Omega(x)|}{|x|^{2}}\right| b(x)-b(y)|| b_{j}(y) \mid \frac{|y|^{1 / 2}}{|x-y|^{3 / 2}} \mathrm{~d} y \\
& \leq C 2^{(j-k) / 2} \int_{B_{j}}\left|\frac{|\Omega(x-y)|}{|x-y|^{2}}-\frac{|\Omega(x)|}{|x|^{2}}\right| b(x)-b(y)|| b_{j}(y) \mid \mathrm{d} y .
\end{aligned}
$$

Similarly, we consider $\Upsilon_{2}$. Noting that $|x-y| \sim|x|$. By the Minkowski's inequality, the generalized Hölder's inequality and the vanishing moments of $b_{j}$ we have

$$
\begin{aligned}
\Upsilon_{2} & \leq C \int_{\mathbb{R}^{n}}\left|\frac{|\Omega(x-y)|}{|x-y|^{n-1}}-\frac{|\Omega(x)|}{|x|^{n-2}}\right| b(x)-b(y)|| b_{j}(y) \mid\left(\int_{|x|}^{\infty} \frac{\mathrm{d} t}{t^{3}}\right)^{1 / 2} \mathrm{~d} y \\
& \leq C \int_{B_{j}}\left|\frac{|\Omega(x-y)|}{|x-y|^{2}}-\frac{|\Omega(x)|}{|x|^{2}}\right| b(x)-b(y)|| b_{j}(y) \mid \mathrm{d} y .
\end{aligned}
$$

So we have

$$
\left|\left[b, \mu_{\Omega}\right]\left(b_{j}\right)(x)\right| \leq C \int_{B_{j}}\left|\frac{|\Omega(x-y)|}{|x-y|^{n}}-\frac{|\Omega(x)|}{|x|^{n}}\right| b(x)-b(y)|| b_{j}(y) \mid \mathrm{d} y .
$$

From lemma 2.10 and the Minkowski's inequality we have

$$
\begin{aligned}
& \left\|\left[b, \mu_{\Omega}\right]\left(b_{j}\right) \chi_{k}\right\|_{L^{p(\cdot)}\left(\mathbb{R}^{n}\right)} \\
& \leq C \int_{B_{j}}\left|\left\|\frac{|\Omega(x-y)|}{|x-y|^{n}}-\frac{|\Omega(x)|}{|x|^{n}}|b(x)-b(y)| \chi_{k}(\cdot)\right\|_{L^{p(\cdot)}\left(\mathbb{R}^{n}\right)}\right| b_{j}(y) \mid \mathrm{d} y \\
& \leq C \int_{B_{j}}||\left|\frac{\Omega(\cdot-y) \mid}{|\cdot-y|^{n}}-\frac{|\Omega(\cdot)|}{|\cdot|^{n} \mid}\right| b(\cdot)-b(0)\left|\chi_{k}(\cdot) \|_{L^{p(\cdot)}\left(\mathbb{R}^{n}\right)}\right| b_{j}(y) \mid \\
& +C \int_{B_{j}}|| \frac{|\Omega(\cdot-y)|}{|\cdot-y|^{n}}-\frac{|\Omega(\cdot)|}{|\cdot|^{n}}\left|\chi_{k}(\cdot) \|_{L^{p(\cdot)}\left(\mathbb{R}^{n}\right)}\right| b(0)-b(y)|| b_{j}(y) \mid \\
& :=\Upsilon_{1}^{*}+\Upsilon_{2}^{*} \text {. }
\end{aligned}
$$
For $\Upsilon_{1}^{*}$, noting $s>p^{\prime}$, we denote $\tilde{p}^{\prime}(\cdot)>1$ and $\frac{1}{p(x)}=\frac{1}{\tilde{p}^{\prime}(x)}+\frac{1}{s}$. By
lemma 2.2 we have

$$
\begin{aligned}
& \left\||| \frac{|\Omega(\cdot-y)|}{|\cdot-y|^{n}}-\frac{|\Omega(\cdot)|}{|\cdot|^{n} \mid}|b(\cdot)-b(0)| \chi_{k}(\cdot)\right\|_{L^{p(\cdot)}\left(\mathbb{R}^{n}\right)} \\
& \leq\left\||| \frac{|\Omega(\cdot-y)|}{|\cdot-y|^{n}}-\frac{|\Omega(\cdot)|}{|\cdot|^{n} \mid} \chi_{k}(\cdot)\right\|_{L^{(\cdot)}\left(\mathbb{R}^{n}\right)}\|b(0)-b(y)\| b_{j}(y) \|_{L^{p^{\prime}(\cdot)}\left(\mathbb{R}^{n}\right)} \\
& \leq C\|b\|_{L i p_{\gamma}} 2^{k \gamma}\left\|\frac{|\Omega(\cdot-y)|}{|\cdot-y|^{n}}-\frac{|\Omega(\cdot)|}{|\cdot|^{n} \mid} \chi_{k}(\cdot)\right\|_{L^{s(\cdot)}\left(\mathbb{R}^{n}\right)}\left\|\chi_{B_{K}}\right\|_{L^{p^{\prime}(\cdot)}\left(\mathbb{R}^{n}\right)} \cdot
\end{aligned}
$$

When $\left|B_{k}\right| \leq 2^{n}$ and $x_{k} \in B_{k}$, by Lemma 2.12 we have 


$$
\left\|\chi_{B_{K}}\right\|_{L^{p^{\prime}(\cdot)}\left(\mathbb{R}^{n}\right)} \simeq\left|B_{k}\right| \frac{1}{p^{\prime}\left(x_{k}\right)} \approx\left\|\chi_{B_{K}}\right\|_{L^{p(\cdot)}\left(\mathbb{R}^{n}\right)}\left|B_{k}\right|^{\frac{-1}{s}-\frac{-\gamma}{n}} .
$$

When $\left|B_{k}\right| \geq 1$ we have

$$
\left.\left\|\chi_{B_{K}}\right\|_{L^{p^{\prime}(\cdot)}\left(\mathbb{R}^{n}\right)} \simeq\left|B_{k} \frac{1}{\left.\right|^{p^{\prime}(\infty)}} \approx\left\|\chi_{B_{K}}\right\|_{L^{p(\cdot)}\left(\mathbb{R}^{n}\right)}\right| B_{k}\right|^{\frac{-1}{s}-\frac{-\gamma}{n}} .
$$

So we obtain

$$
\left\|\chi_{B_{K}}\right\|_{L^{p^{\prime}(\cdot)}\left(\mathbb{R}^{n}\right)} \approx\left\|\chi_{B_{K}}\right\|_{L^{p(\cdot)}\left(\mathbb{R}^{n}\right)}\left|B_{k}\right|^{\frac{-1}{s}-\frac{-\gamma}{n}} .
$$

By lemma 2.9 we have

$$
\begin{aligned}
& || \frac{|\Omega(\cdot-y)|}{|\cdot-y|}-\frac{|\Omega(\cdot)|}{|\cdot|^{n}} \mid \chi_{k}(\cdot) \|_{L^{(\cdot)}\left(\mathbb{R}^{n}\right)} \\
& \leq 2^{(k-1)\left(\frac{n}{s}-n\right)}\left\{\frac{|y|}{2^{k}}+\int_{\frac{|y|}{2^{k}}}^{\frac{|y|}{2^{k-1}}} \frac{\omega_{s}(\delta)}{\delta} \mathrm{d} \delta\right\} \\
& \leq 2^{(k-1)\left(\frac{n}{s}-n\right)}\left\{2^{j-k+1}+2^{(j-k+1) \gamma} \int_{0}^{1} \frac{\omega_{s}(\delta)}{\delta} \mathrm{d} \delta\right\} \\
& \leq 2^{(k-1)\left(\frac{n}{s}-n\right)} 2^{(j-k) \gamma} .
\end{aligned}
$$

Now, by using the generalized Hölder's inequality we get:

$$
\begin{aligned}
\Upsilon_{1}^{*} & \leq \int_{B_{j}}\left\||| \frac{\Omega(\cdot-y) \mid}{|\cdot-y|^{n}}-\frac{\mid \Omega(\cdot)}{|\cdot|^{n}}|| b(\cdot)-b(y)\left|\chi_{k}(\cdot) \|_{L^{(\cdot)}\left(\mathbb{R}^{n}\right)}\right| b_{j}(y) \mid \mathrm{d} y\right. \\
& \leq C\|b\|_{L i p_{\gamma}} 2^{-k n+(j-k) \gamma-k \gamma}\left\|\chi_{B_{K}}\right\|_{L^{p(\cdot)}\left(\mathbb{R}^{n}\right)}\left|B_{k}\right|^{\frac{-1}{s}-\frac{-\gamma}{n}} \int_{B_{j}}\left|b_{j}(y)\right| \mathrm{d} y \\
& \leq C\|b\|_{L i p_{\gamma}} 2^{-k n+(j-k) \gamma-k \gamma}\left\|\chi_{B_{K}}\right\|_{L^{p(\cdot)}\left(\mathbb{R}^{n}\right)}\left\|b_{j}\right\|_{L^{p(\cdot)}\left(\mathbb{R}^{n}\right)}\left\|\chi_{B_{j}}\right\|_{L^{p^{\prime}(\cdot)}\left(\mathbb{R}^{n}\right)} .
\end{aligned}
$$

For $\Upsilon_{2}^{*}$ similar to the method of $\Upsilon_{1}^{*}$ we have

$$
\begin{aligned}
& \left\|\frac{|\Omega(\cdot-y)|}{|\cdot-y|^{n}}-\frac{|\Omega(\cdot)|}{|\cdot|^{n} \mid} \chi_{k}(\cdot)\right\|_{L^{p(\cdot)}\left(\mathbb{R}^{n}\right)} \\
& \leq\left\|\left|\frac{|\Omega(\cdot-y)|}{|\cdot-y|^{n}}-\frac{|\Omega(\cdot)|}{|\cdot|^{n}}\right| \chi_{k}(\cdot)\right\|_{L^{(\cdot)}\left(\mathbb{R}^{n}\right)}\left\|\chi_{k}(\cdot)\right\|_{L^{p^{(\cdot)}()}\left(\mathbb{R}^{n}\right)} \\
& \leq|| \frac{|\Omega(\cdot-y)|}{|\cdot-y|^{n}}-\frac{|\Omega(\cdot)|}{|\cdot|^{n} \mid} \chi_{k}(\cdot)\left\|_{L^{5(\cdot)}\left(\mathbb{R}^{n}\right)}\right\| \chi_{B_{k}}(\cdot) \|_{L^{p^{\prime}(\cdot)}\left(\mathbb{R}^{n}\right)} \\
& \leq 2^{(k-1)\left(\frac{n}{s}-n\right)} 2^{(j-k) \gamma}\left\|\chi_{B_{K}}\right\|_{L^{p^{\prime}(\cdot)}\left(\mathbb{R}^{n}\right)} \\
& \leq 2^{-k n+(j-k) \gamma-k \gamma}\left\|\chi_{B_{k}}\right\|_{L^{p()}\left(\mathbb{R}^{n}\right)} .
\end{aligned}
$$

Now, by using the generalized Hölder's inequality we get: 


$$
\begin{aligned}
& \Upsilon_{2}^{*} \leq \int_{B_{j}}\left\|\frac{|\Omega(\cdot-y)|}{|\cdot-y|^{n}}-\frac{|\Omega(\cdot)|}{|\cdot|^{n}}\left|\chi_{k}(\cdot) \|_{L^{(\cdot)}\left(\mathbb{R}^{n}\right)}\right| b(0)-b(y)|| b_{j}(y) \mid \mathrm{d} y\right. \\
& \leq C\|b\|_{L i p_{\gamma}} 2^{-k n+2(j-k) \gamma}\left\|\chi_{B_{k}}\right\|_{L^{p^{(\cdot)}\left(\mathbb{R}^{n}\right)}}\left\|b_{j}\right\|_{L^{p(\cdot)}\left(\mathbb{R}^{n}\right)}\left\|\chi_{B_{j}}\right\|_{L^{p^{(\cdot)}\left(\mathbb{R}^{n}\right)}} \\
& \leq C\|b\|_{L i p_{\gamma}} 2^{-k n+(j-k) \gamma}\left\|\chi_{B_{k}}\right\|_{L^{p(\cdot)}\left(\mathbb{R}^{n}\right)}\left\|b_{j}\right\|_{L^{p(\cdot)}\left(\mathbb{R}^{n}\right)}\left\|\chi_{B_{j}}\right\|_{L^{p^{\prime}(\cdot)}\left(\mathbb{R}^{n}\right)} \text {. }
\end{aligned}
$$

Now by (3.3), (3.4), and lemmas 2.5 and 2.6, we have

$$
\begin{aligned}
& \left\|\left[b, \mu_{\Omega}\right]\left(b_{j}\right) \chi_{k}\right\|_{L^{p(\cdot)}\left(\mathbb{R}^{n}\right)} \\
& \leq C\|b\|_{L i p_{\gamma}} 2^{-k n+(j-k) \gamma}\left\|\chi_{B_{k}}\right\|_{L^{p(\cdot)}\left(\mathbb{R}^{n}\right)}\left\|b_{j}\right\|_{L^{p(\cdot)}\left(\mathbb{R}^{n}\right)}\left\|\chi_{B_{j}}\right\|_{L^{p^{\prime}(\cdot)}\left(\mathbb{R}^{n}\right)} \\
& \leq C\|b\|_{L i p_{\gamma}} 2^{(j-k) \gamma}\left\|b_{j}\right\|_{L^{p^{(\cdot)}\left(\mathbb{R}^{n}\right)}} \frac{\left\|\chi_{B_{j}}\right\|_{L^{p^{(}()}\left(\mathbb{R}^{n}\right)}}{\left\|\chi_{B_{k}}\right\|_{L^{p^{(}(\cdot)}\left(\mathbb{R}^{n}\right)}} \\
& \leq C 2^{-j \alpha+(j-k)\left(\gamma+n \delta_{2}\right)}\|b\|_{L i p_{\gamma}} .
\end{aligned}
$$

Firstly we estimate $I$. We need to show that there exists a positive constant $C$, such that $I \leq C \Delta$, we consider

$$
\begin{aligned}
I= & \sup _{L \geq 0, L \in \mathbb{Z}} 2^{-L \lambda q} \sum_{k=-\infty}^{L} 2^{k q \alpha(0)}\left|\lambda_{j}\right|\left\|\left[b, \mu_{\Omega}\right](f) \chi_{k}\right\|_{L^{D^{(\cdot)}}}^{q}\left(\mathbb{R}^{n}\right) \\
\leq & \sup _{L \geq 0, L \in \mathbb{Z}} 2^{-L \lambda q} \sum_{k=-\infty}^{L} 2^{k q \alpha(0)}\left(\sum_{j=K}^{\infty}\left|\lambda_{j}\right|\left\|\left[b, \mu_{\Omega}\right] \chi_{k}\right\|_{L^{P(\cdot)}\left(\mathbb{R}^{n}\right)}\right)^{q} \\
& +\sup _{L \geq 0, L \in \mathbb{Z}} 2^{-L \lambda q} \sum_{k=-\infty}^{L} 2^{k q \alpha(0)}\left(\sum_{j=-\infty}^{k-1}\left|\lambda_{j}\right|\left\|\left[b, \mu_{\Omega}\right] \chi_{k}\right\|_{L^{P(\cdot)}\left(\mathbb{R}^{n}\right)}\right)^{q} \\
:= & I_{1}+I_{2} .
\end{aligned}
$$

By the $\left(L^{p(\cdot)}\left(\mathbb{R}^{n}\right), L^{q(\cdot)}\left(\mathbb{R}^{n}\right)\right)$, bounbedness of the commutator $\left[b, \mu_{\Omega}\right]$ on $L^{p(\cdot)}$ (see [15]), we have the following. Therefore, when $0<q \leq 1$

$$
\begin{aligned}
I_{1}= & \left.\left.\sup _{L \geq 0, L \in \mathbb{Z}} 2^{-L \lambda q} \sum_{k=-\infty}^{L} 2^{k q \alpha(0)}\left(\sum_{j=K}^{\infty}|| b, \mu_{\Omega}\right] \chi_{k}\right|_{L^{p(\cdot)}\left(\mathbb{R}^{n}\right)}\right)^{q} \\
\leq & \sup _{L \leq 0, L \in \mathbb{Z}} 2^{-L \lambda q} \sum_{k=-\infty}^{L} 2^{k q \alpha(0)}\left(\sum_{j=k}^{\infty}\left|\lambda_{j}\right| 2^{-j \alpha_{j}}\right)^{q} \\
\leq & \sup _{L \leq 0, L \in \mathbb{Z}} 2^{-L \lambda q} \sum_{k=-\infty}^{L} 2^{k q \alpha(0)}\left(\sum_{j=k}^{-1}\left|\lambda_{j}\right| 2^{-j \alpha(0) j q}+\sum_{j=0}^{\infty}\left|\lambda_{j}\right| 2^{-j \alpha_{\infty} q}\right) \\
\leq & \sup _{L \leq 0, L \in \mathbb{Z}} 2^{-L \lambda q} \sum_{k=-\infty}^{L} \sum_{j=k}^{-1}\left|\lambda_{j}\right|^{q} 2^{\alpha(0)(k-j) q} \\
& +\sup _{L \leq 0, L \in \mathbb{Z}} 2^{-L \lambda q} \sum_{k=-\infty}^{L} 2^{k q \alpha(0)} \sum_{j=0}^{\infty}\left|\lambda_{j}\right|^{q} 2^{-j \alpha_{\infty} q} \\
\leq & \sup _{L \leq 0, L \in \mathbb{Z}} 2^{-L \lambda q} \sum_{j=-\infty}^{-1}\left|\lambda_{j}\right|^{q} \sum_{k=-\infty}^{j} 2^{\alpha(0)(k-j) q} \\
& +\sup _{L \leq 0, L \in \mathbb{Z} j=0}^{\infty} 2^{-j \lambda q}\left|\lambda_{j}\right|^{q} 2^{\left(\lambda-\alpha_{\infty}\right) j q} 2^{-L \lambda q} \sum_{k=-\infty}^{L} 2^{k q \alpha(0)}
\end{aligned}
$$




$$
\begin{aligned}
& \leq \sup _{L \leq 0, L \in \mathbb{Z}} 2^{-L \lambda q} \sum_{j=-\infty}^{L}\left|\lambda_{j}\right|^{q}+\sup _{L \leq 0, L \in \mathbb{Z}} 2^{-L \lambda q} \sum_{j=L}^{-1}\left|\lambda_{j}\right|^{q} \sum_{k=-\infty}^{j} 2^{\alpha(0)(k-j) q} \\
& +\Delta \sup _{L \leq 0, L \in \mathbb{Z} j=0} \sum^{\infty} 2^{\left(\lambda-\alpha_{\infty}\right) j q} \sum_{k=-\infty}^{L} 2^{(\alpha(0) k-L \lambda) q} \\
& \leq \Delta+\sup _{L \leq 0, L \in \mathbb{Z} j=L} \sum^{-1} 2^{-j \lambda q}\left|\lambda_{j}\right|^{q} 2^{(j-L) \lambda q} \sum_{k=-\infty}^{j} 2^{\alpha(0)(k-j) q}+\Delta \\
& \leq \Delta+\sup _{L \leq 0, L \in \mathbb{Z}_{j=L}} 2^{-j \lambda q}\left|\lambda_{j}\right|^{q} 2^{(j-L) \lambda q} \sum_{k=-\infty}^{j} 2^{\alpha(0)(k-j) q} \\
& \leq \Delta .
\end{aligned}
$$

When $0<q<\infty$, let $1 / q+1 / q^{\prime}=1$ we have

$$
\begin{aligned}
& I_{1}=\sup _{L \geq 0, L \in \mathbb{Z}} 2^{-L \lambda q} \sum_{k=-\infty}^{L} 2^{k q \alpha(0)}\left(\sum_{j=K}^{\infty}\left|\lambda_{j}\right|\left\|\left[b, \mu_{\Omega}\right] \chi_{k}\right\|_{L^{p(\cdot)}\left(\mathbb{R}^{n}\right)}\right)^{q} \\
& \leq \sup _{L \leq 0, L \in \mathbb{Z}} 2^{-L \lambda q} \sum_{k=-\infty}^{L} 2^{k q \alpha(0)}\left(\sum_{j=k}^{\infty}\left|\lambda_{j}\right| 2^{-j \alpha}\right)^{q} \\
& \leq \sup _{L \leq 0, L \in \mathbb{Z}} 2^{-L \lambda q} \sum_{k=-\infty}^{L}\left(\sum_{j=k}^{-1}\left|\lambda_{j}\right| 2^{\alpha(0)(k-j)}\right)^{q} \\
& +\sup _{L \leq 0, L \in \mathbb{Z}} 2^{-L \lambda q} \sum_{k=-\infty}^{L} 2^{k q \alpha(0)}\left(\sum_{j=0}^{\infty}\left|\lambda_{j}\right| 2^{-j \alpha_{\infty}}\right)^{q} \\
& \leq \sup _{L \leq 0, L \in \mathbb{Z}} 2^{-L \lambda q} \sum_{k=-\infty}^{L}\left(\sum_{j=k}^{-1}\left|\lambda_{j}\right|^{q} 2^{\alpha(0)(k-j) q / 2}\right)\left(\sum_{j=k}^{-1} 2^{\alpha(0)(k-j) q^{\prime} / 2}\right)^{q / q^{\prime}} \\
& +\sup _{L \leq 0, L \in \mathbb{Z}} 2^{-L \lambda q} \sum_{k=-\infty}^{L} 2^{k q \alpha(0)}\left(\sum_{j=0}^{\infty}\left|\lambda_{j}\right|^{q} 2^{-j \alpha_{\infty} q / 2}\right)\left(\sum_{j=0}^{\infty} 2^{-j \alpha_{\infty} q^{\prime} / 2}\right)^{q / q^{\prime}} \\
& \leq \sup _{L \leq 0, L \in \mathbb{Z}} 2^{-L \lambda q} \sum_{k=-\infty}^{L} \sum_{j=k}^{-1}\left|\lambda_{j}\right|^{q} 2^{(j-k) q / 2} \\
& +\sup _{L \leq 0, L \in \mathbb{Z}} 2^{-L \lambda q} \sum_{k=-\infty}^{L} 2^{k q \alpha(0)} \sum_{j=0}^{\infty}\left|\lambda_{j}\right|^{q} 2^{-j \alpha_{\infty} q / 2} \\
& \leq \sup _{L \leq 0, L \in \mathbb{Z}} 2^{-L \lambda q} \sum_{j=-\infty}^{-1}\left|\lambda_{j}\right|^{q} \sum_{k=-\infty}^{j} 2^{\alpha(0)(j-k) q / 2} \\
& +\sup _{L \leq 0, L \in \mathbb{Z}} \sum_{j=0}^{\infty} 2^{j \lambda q}\left|\lambda_{j}\right|^{q} 2^{\left(\lambda-\alpha_{\infty} / 2\right) j q} 2^{-L \lambda q} \sum_{k=-\infty}^{L} 2^{k q \alpha(0)} \\
& \leq \sup _{L \leq 0, L \in \mathbb{Z}} 2^{-L \lambda q} \sum_{j=-\infty}^{L}\left|\lambda_{j}\right|^{q}+\sup _{L \leq 0, L \in \mathbb{Z}} 2^{-L \lambda q} \sum_{j=L}^{-1}\left|\lambda_{j}\right|^{q} \sum_{j=-\infty}^{j} 2^{\alpha(0)(k-j) q / 2} \\
& +\Delta \sup _{L \leq 0, L \in \mathbb{Z}} \sum_{j=0}^{\infty} 2^{\left(\lambda-\alpha_{\infty} / 2\right) j / 2 q} \sum_{k=-\infty}^{L} 2^{k q \alpha(0)-L \lambda q} \\
& \leq \Delta+\sup _{L \leq 0, L \in \mathbb{Z}} \sum_{j=L}^{-1} 2^{-j \lambda q}\left|\lambda_{j}\right|^{q} 2^{(j-L) \lambda q} \sum_{j=-\infty}^{j} 2^{\alpha(0)(k-j) q / 2}+\Delta \\
& \leq \Delta+\Delta \sup _{L \leq 0, L \in \mathbb{Z}} \sum_{j=L}^{-1} 2^{(j-L) \lambda q} \sum_{j=-\infty}^{j} 2^{\alpha(0)(k-j) q / 2}
\end{aligned}
$$

We estimate $I_{2}$ by lemma 2.1 when $0<q \leq 1$ by $n \delta_{2} \leq \alpha(0)<\gamma+n \delta_{2}$, we get 


$$
\begin{aligned}
I_{2} & =\sup _{L \geq 0, L \in \mathbb{Z}} 2^{-L \lambda q} \sum_{k=-\infty}^{L} 2^{k q \alpha(0)}\left(\sum_{j=-\infty}^{k-1}\left|\lambda_{j}\right|\left\|\left[b, \mu_{\Omega}\right] \chi_{k}\right\|_{L^{p(\cdot)}\left(\mathbb{R}^{n}\right)}\right)^{q} \\
& \leq C\|b\|_{L i p_{\gamma}}^{q} \sup _{L \geq 0, L \in \mathbb{Z}} 2^{-L \lambda q} \sum_{k=-\infty}^{L} 2^{k q \alpha(0)}\left(\sum_{j=-\infty}^{k-1} 2^{-j \alpha+(j-k)\left(\gamma+n \delta_{2}\right)}\left|\lambda_{j}\right|\right)^{p} \\
& \leq C\|b\|_{L i p_{\gamma}}^{q} \sup _{L \geq 0, L \in \mathbb{Z}} 2^{-L \lambda q} \sum_{k=-\infty}^{L} 2^{k q \alpha(0)}\left(\sum_{j=-\infty}^{k-1}\left|\lambda_{j}\right|^{p} 2^{\left(-j \alpha+(j-k)\left(\gamma+n \delta_{2}\right)\right) q}\right) \\
& \leq C\|b\|_{L i p_{\gamma}}^{q} \sup _{L \geq 0, L \in \mathbb{Z}} 2^{-L \lambda q} \sum_{j=-\infty}^{L}\left|\lambda_{j}\right|^{p} \sum_{k=j+1}^{-1} 2^{q(j-k)\left[\gamma+n \delta_{2}-\alpha(0)\right]} \\
& \leq C\|b\|_{L i p_{\gamma}}^{q} \Delta .
\end{aligned}
$$

When $0<q<\infty$, let $1 / q+1 / q^{\prime}=1$. Since $n \delta_{2} \leq \alpha(0)<\gamma+n \delta_{2}$, by Hölder's inequality, we have

$$
\begin{aligned}
I_{2}= & \sup _{L \geq 0, L \in \mathbb{Z}} 2^{-L \lambda q} \sum_{k=-\infty}^{L} 2^{k q \alpha(0)}\left(\sum_{j=-\infty}^{k-1}|\lambda|\left\|\left[b, \mu_{\Omega}\right] \chi_{k}\right\|_{L^{p(\cdot)}\left(\mathbb{R}^{n}\right)}\right)^{q} \\
\leq & C\|b\|_{L i p_{\gamma}}^{q} \sup _{L \geq 0, L \in \mathbb{Z}} 2^{-L \lambda q} \sum_{k=-\infty}^{L} 2^{k q \alpha(0)}\left(\sum_{j=-\infty}^{k-1} 2^{-j \alpha+(j-k)\left(\gamma+n \delta_{2}\right)}\left|\lambda_{j}\right|\right)^{p} \\
\leq & C\|b\|_{L i p_{\gamma}}^{q} \sup _{L \geq 0, L \in \mathbb{Z}} 2^{-L \lambda q} \sum_{k=-\infty}^{L} 2^{k q \alpha(0)}\left(\sum_{j=-\infty}^{k-1}\left|\lambda_{j}\right|^{p} 2^{\left(-j \alpha+(j-k)\left(\gamma+n \delta_{2}\right)\right) q / 2}\right) \\
& \times\left(\sum_{j=-\infty}^{k-1} 2^{\left(-j \alpha+(j-k)\left(\gamma+n \delta_{2}\right)\right) q^{\prime} / 2}\right)^{q / q^{\prime}} \\
\leq & C\|b\|_{L i p_{\gamma}}^{q} \sup _{L \geq 0, L \in \mathbb{Z}} 2^{-L \lambda q} \sum_{k=-\infty}^{L} 2^{k q \alpha(0)}\left(\sum_{j=-\infty}^{k-1}\left|\lambda_{j}\right|^{p} 2^{\left(-j \alpha+(j-k)\left(\gamma+n \delta_{2}\right)\right) q / 2}\right) \\
\leq & C\|b\|_{L i p_{\gamma}}^{q} \sup _{L \geq 0, L \in \mathbb{Z}} 2^{-L \lambda q} \sum_{j=-\infty}^{L}\left|\lambda_{j}\right|^{p} \sum_{k=j+1}^{-1} 2^{q / 2(j-k)\left[\gamma+n \delta_{2}-\alpha(0)\right]} \\
\leq & C\|b\|_{L i p_{\gamma}}^{q} \Delta .
\end{aligned}
$$

Secondly we estimate II . We need to show that there exists a positive constant $C$, such that $I I \leq C \Delta$, we consider

$$
\begin{aligned}
I I= & \sum_{k=-\infty}^{-1} 2^{k q \alpha(0)}\left\|\left[b, \mu_{\Omega}\right](f) \chi_{k}\right\|_{L^{p(\cdot)}}^{q} \\
\leq & \sum_{k=-\infty}^{-1} 2^{k q \alpha(0)}\left(\sum_{j=k}^{\infty}\left|\lambda_{j}\right|\left\|\left[b, \mu_{\Omega}\right]\left(b_{j}\right) \chi_{k}\right\|_{L^{p(\cdot)}}\right)^{q} \\
& +\sum_{k=-\infty}^{-1} 2^{k q \alpha(0)}\left(\sum_{j=-\infty}^{k-1}\left|\lambda_{j}\right|\left\|\left[b, \mu_{\Omega}\right]\left(b_{j}\right) \chi_{k}\right\|_{L^{p(\cdot)}}\right)^{q} \\
:= & I I_{1}+I I_{2} .
\end{aligned}
$$

When $0<q \leq 1$, we get

$$
\begin{aligned}
I_{1} & =\sum_{k=-\infty}^{-1} 2^{k q \alpha(0)}\left(\sum_{j=k}^{\infty}\left|\lambda_{j}\right|\left\|\left[b, \mu_{\Omega}\right]\left(b_{j}\right) \chi_{k}\right\|_{L^{p(\cdot)}}\right)^{q} \\
& \leq \sum_{k=-\infty}^{-1} 2^{k q \alpha(0)}\left(\sum_{j=-\infty}^{k-1} 2^{-j \alpha_{j}}\left|\lambda_{j}\right|\right)^{p}
\end{aligned}
$$




$$
\begin{aligned}
& \leq \sum_{k=-\infty}^{-1} 2^{k q \alpha(0)}\left(\sum_{j=k}^{-1}\left|\lambda_{j}\right|^{q} 2^{-j q \alpha(0)}+\sum_{j=0}^{\infty}\left|\lambda_{j}\right|^{q} 2^{-j q \alpha_{\infty}}\right) \\
& \leq \sum_{k=-\infty}^{-1} \sum_{j=k}^{-1}\left|\lambda_{j}\right|^{q} 2^{\alpha(0)(k-j) q}+\sum_{k=-\infty}^{-1} 2^{k q \alpha(0)} \sum_{j=0}^{\infty}\left|\lambda_{j}\right|^{q} 2^{-j \alpha_{\infty} q} \\
& \leq \sum_{k=-\infty}^{-1}\left|\lambda_{j}\right|^{q} \sum_{k=\infty}^{j} 2^{q \alpha(0)(k-j)}+\sum_{j=0}^{\infty}\left|\lambda_{j}\right|^{q} 2^{-j q \alpha_{\infty}} \sum_{k=-\infty}^{-1} 2^{k q \alpha(0)} \\
& \leq \sum_{k=-\infty}^{-1}\left|\lambda_{j}\right|^{q}+\sum_{j=0}^{\infty} 2^{-j \lambda q}\left|\lambda_{j}\right|^{q} 2^{-j q \alpha_{\infty}} \sum_{k=-\infty}^{-1} 2^{k q \alpha(0)} \\
& \leq \Delta+\Delta \sum_{i=-\infty}^{j}\left|\lambda_{i}\right|^{q} \sum_{j=0}^{\infty} 2^{\left(\lambda-\alpha_{\infty}\right) j q} \sum_{k=-\infty}^{j} 2^{k q \alpha(0)} \\
& \leq \Delta .
\end{aligned}
$$

When $0<q<\infty$, let $1 / q_{1}+1 / q_{1}^{\prime}=1$ we have

$$
\begin{aligned}
I_{1} & =\sum_{k=-\infty}^{-1} 2^{k q \alpha(0)}\left(\left.\sum_{j=k}^{\infty}\left|\lambda_{j}\right|||\left[b, \mu_{\Omega}\right]\left(b_{j}\right) \chi_{k}\right|_{L^{p(-)}}\right)^{q} \\
\leq & \sum_{k=-\infty}^{-1} 2^{k q \alpha(0)}\left(\sum_{j=k}^{\infty}\left|\lambda_{j}\right| 2^{-j \alpha}\right)^{q} \\
\leq & \sum_{k=-\infty}^{-1}\left(\sum_{j=k}^{-1}\left|\lambda_{j}\right| 2^{\alpha(0)(j-k)}\right)^{q}+\sum_{k=-\infty}^{-1} 2^{k q \alpha(0)}\left(\sum_{j=0}^{\infty}\left|\lambda_{j}\right| 2^{-j \alpha_{\infty}}\right)^{q} \\
\leq & \sum_{k=-\infty}^{-1}\left(\sum_{j=k}^{-1}\left|\lambda_{j}\right|^{q} 2^{q / 2 \alpha(0)(j-k)}\right)\left(\sum_{j=k}^{-1} 2^{\alpha(0)(j-k) q^{\prime} / 2}\right)^{q / q^{\prime}} \\
& +\sum_{k=-\infty}^{-1} 2^{k q \alpha(0)}\left(\sum_{j=0}^{\infty}\left|\lambda_{j}\right|^{q} 2^{-q / 2 j \alpha_{\infty}}\right)\left(\sum_{j=0}^{\infty} 2^{-q^{\prime} / 2 j \alpha_{\infty}}\right)^{q / q^{\prime}} \\
\leq & \sum_{k=-\infty}^{-1}\left|\lambda_{j}\right|^{q} \sum_{k=-\infty}^{j} 2^{q / 2 \alpha(0)(j-k)}+\sum_{k=-\infty}^{-1} 2^{k q \alpha(0)} \sum_{j=0}^{\infty}\left|\lambda_{j}\right|^{q} 2^{-q / 2 j \alpha_{\infty}} \\
\leq & \sum_{k=-\infty}^{-1}\left|\lambda_{j}\right|^{q}+\sum_{j=0}^{\infty} 2^{\left(\lambda-\alpha_{\infty} / 2\right) j q} 2^{-\lambda j q} \sum_{i=-\infty}^{j}\left|\lambda_{i}\right|^{q} \sum_{k=-\infty}^{-1} 2^{k q \alpha(0)} \\
\leq & \Delta+\Delta \sum_{j=0}^{\infty} 2^{\left(\lambda-\alpha_{\infty} / 2\right) j q} \sum_{k=-\infty}^{-1} 2^{k q \alpha(0)} \\
\leq & \Delta .
\end{aligned}
$$

For $I I_{2}$, when $0<q \leq 1$, by $n \delta_{2} \leq \alpha(0)<\gamma+n \delta_{2}$ we get

$$
\begin{aligned}
I I_{2} & =\sum_{k=-\infty}^{-1} 2^{k q \alpha(0)}\left(\sum_{j=\infty}^{k-1}\left|\lambda_{j}\right|\left\|\left[b, \mu_{\Omega}\right]\left(b_{j}\right) \chi_{k}\right\|_{L^{p(\cdot)}}\right)^{q} \\
& \leq \sum_{k=-\infty}^{-1} 2^{k q \alpha(0)}\left(C\|b\|_{L i p_{\gamma}} \sum_{j=\infty}^{k-1}\left|\lambda_{j}\right| 2^{-j \alpha+(j-k)\left(\gamma+n \delta_{2}\right)}\right)^{q} \\
& \leq C\|b\|_{L i p_{\gamma}}^{q} \sum_{k=-\infty}^{-1} 2^{k q \alpha(0)}\left(\sum_{j=\infty}^{k-1}\left|\lambda_{j}\right|^{q} 2^{\left[-j \alpha+(j-k)\left(\gamma+n \delta_{2}\right)\right] q}\right) \\
& \leq C\|b\|_{L i p_{\gamma}}^{q} \sum_{j=-\infty}^{-1}\left|\lambda_{j}\right|^{q} \sum_{k=j+1}^{-1} 2^{\left[-j \alpha_{\infty}+(j-k)\left(\gamma+n \delta_{2}\right)\right] q} \\
& \leq C\|b\|_{L i p_{\gamma}}^{q} \Delta .
\end{aligned}
$$


When $1<q<\infty$, let $1 / q+1 / q^{\prime}=1$. Since $n \delta_{2} \leq \alpha(0)<\gamma+n \delta_{2}$, by Hölder's inequality, we have

$$
\begin{aligned}
I I_{2} & =\sum_{k=-\infty}^{-1} 2^{k q \alpha(0)}\left(\sum_{j=\infty}^{k-1}\left|\lambda_{j}\right|\left\|\left[b, \mu_{\Omega}\right]\left(b_{j}\right) \chi_{k}\right\|_{L^{p \cdot()}}\right)^{q} \\
\leq & \sum_{k=-\infty}^{-1} 2^{k q \alpha(0)}\left(C\|b\|_{L i p_{\gamma}} \sum_{j=\infty}^{k-1}\left|\lambda_{j}\right|^{-j \alpha+(j-k)\left(\gamma+n \delta_{2}\right)}\right)^{q} \\
\leq & C\|b\|_{L i p_{\gamma}}^{q} \sum_{k=-\infty}^{-1} 2^{k q \alpha(0)}\left(\sum_{j=\infty}^{k-1}\left|\lambda_{j}\right|^{q} 2^{\left[-j \alpha+(j-k)\left(\gamma+n \delta_{2}\right)\right] q / 2}\right) \\
& \times\left(\sum_{j=\infty}^{k-1}\left|\lambda_{j}\right|^{q} 2^{\left[-j \alpha+(j-k)\left(\gamma+n \delta_{2}\right)\right] q^{\prime / 2}}\right)^{q / q^{\prime}} \\
\leq & C \|\left. b\right|_{L i p_{\gamma}} ^{q} \sum_{k=-\infty}^{-1} 2^{k q \alpha(0)}\left(\sum_{j=-\infty}^{k-1}\left|\lambda_{j}\right|^{q} 2^{\left[-j \alpha+(j-k)\left(\gamma+n \delta_{2}\right)\right] q^{\prime} / 2}\right) \\
\leq & C\|b\|_{L i p_{\gamma}}^{q} \sum_{j=-\infty}^{-1}\left|\lambda_{j}\right|^{q} \sum_{k=j+1}^{-1} 2^{(j-k)\left[\gamma+n \delta_{2}-\alpha(0)\right] q / 2} \\
\leq & C\|b\|_{L i p_{\gamma}}^{q} \Delta .
\end{aligned}
$$

Thirdly, we estimate $I I I$, we need to show that there exists a positive constant $C$, such that $I I I \leq C \Delta$

$$
\begin{aligned}
I I I= & \sup _{L \leq 0, L \in \mathbb{Z}} 2^{-L \lambda q} \sum_{k=0}^{L} 2^{k q \alpha(\infty)}\left\|\left[b, \mu_{\Omega}\right](f) \chi_{k}\right\|_{L^{p(\cdot)}}^{q} \\
\leq & \sup _{L \leq 0, L \in \mathbb{Z}} 2^{-L \lambda q} \sum_{k=0}^{L} 2^{k q \alpha(\infty)}\left(\sum_{j=k}^{\infty}\left|\lambda_{j}\right|\left\|\left[b, \mu_{\Omega}\right]\left(b_{j}\right) \chi_{k}\right\|_{L^{p \cdot()}}\right)^{q} \\
& +\sup _{L \leq 0, L \in \mathbb{Z}} 2^{-L \lambda q} \sum_{k=0}^{L} 2^{k q \alpha(\infty)}\left(\sum_{j=\infty}^{k-1}\left|\lambda_{j}\right|\left\|\left[b, \mu_{\Omega}\right] L^{p(\cdot)} \chi_{k}\right\|_{L^{p(\cdot)}}\right)^{q} \\
: & =I I I_{1}+I I I_{2} .
\end{aligned}
$$

When $0<q \leq 1$, by the boundedness of $\left[b, \mu_{\Omega}\right]$ in $L^{p(\cdot)}$ ([20]), we have

$$
\begin{aligned}
& I I I_{1}=\sup _{L \leq 0, L \in \mathbb{Z}} 2^{-L \lambda q} \sum_{k=0}^{L} 2^{k q \alpha_{\infty}}\left(\sum_{j=k}^{\infty}\left|\lambda_{j}\right|\left\|\left[b, \mu_{\Omega}\right]\left(b_{j}\right) \chi_{k}\right\|_{\left.L^{(P)}\right)}\right)^{q} \\
& \leq \sup _{L \leq 0, L \in \mathbb{Z}} 2^{-L \lambda q} \sum_{k=0}^{L} 2^{k q \alpha \alpha_{\infty}} \sum_{j=k}^{\infty}\left|\lambda_{j}\right|^{q}\left\|\left[b, \mu_{\Omega}\right]\left(b_{j}\right) \chi_{k}\right\|_{L^{p()}}^{q} \\
& \leq \sup _{L \leq 0, L \in \mathbb{Z}} 2^{-L \lambda q} \sum_{k=0}^{L} 2^{k q \alpha \alpha_{\infty}} \sum_{j=k}^{\infty}\left|\lambda_{j}\right|^{q} 2^{-\alpha_{j} j q} \\
& \leq \sup _{L \leq 0, L \in \mathbb{Z}} 2^{-L \lambda q} \sum_{k=0}^{L} 2^{k q \alpha_{\infty}} \sum_{j=k}^{\infty}\left|\lambda_{j}\right|^{q} 2^{-\alpha_{\infty} j q} \\
& =\sup _{L \leq 0, L \in \mathbb{Z}} 2^{-L \lambda q} \sum_{j=0}^{L}\left|\lambda_{j}\right|^{q} \sum_{k=0}^{j} 2^{\alpha_{\infty}(k-j) q}+\sup _{L \leq 0, L \in \mathbb{Z}} 2^{-L \lambda q} \sum_{j=L}^{\infty}\left|\lambda_{j}\right|^{q} \sum_{k=0}^{L} 2^{\alpha_{\infty}(k-j) q} \\
& \leq \sup _{L \leq 0, L \in \mathbb{Z}} 2^{-L \lambda q} \sum_{k=0}^{L}\left|\lambda_{j}\right|^{q}+\sup _{L \leq 0, L \in \mathbb{Z} j=L} \sum^{\infty} 2^{(j-L) q \lambda} 2^{-j \lambda q} \sum_{i=-\infty}^{j}\left|\lambda_{i}\right|^{q} \sum_{k=0}^{L} 2^{\alpha_{\infty}(k-j) q} \\
& \leq \Delta+\Delta \sup _{L \leq 0, L \in \mathbb{Z}} \sum_{j=L}^{\infty} 2^{(j-L) q \lambda} 2^{-j \lambda q} 2^{\alpha_{\infty}(L-j) q} \\
& \leq \Delta+\Delta \sup _{L \leq 0, L \in \mathbb{Z}} \sum_{j=L}^{\infty} 2^{(j-L) q\left(\lambda-\alpha_{\infty}\right)}
\end{aligned}
$$

$\leq \Delta$. 
When $0<q \leq \infty$, by $n \delta_{2} \leq \alpha(0), \alpha_{\infty}<\gamma+n \delta_{2}$ and the boundedness of $\left[b, \mu_{\Omega}\right]$ in $L^{p(\cdot)}([20])$ and Hölder's inequality, we get

$$
\begin{aligned}
& I I I_{1}=\sup _{L \leq 0, L \in \mathbb{Z}} 2^{-L \lambda q} \sum_{k=0}^{L} 2^{k q \alpha(\infty)}\left(\sum_{j=k}^{\infty}\left|\lambda_{j}\right|\left\|\left[b, \mu_{\Omega}\right]\left(b_{j}\right) \chi_{k}\right\|_{L^{p(\cdot)}}\right)^{q} \\
& \leq \sup _{L \leq 0, L \in \mathbb{Z}} 2^{-L \lambda q} \sum_{k=0}^{L} 2^{k q \alpha_{\infty}}\left(\left.\sum_{j=k}^{\infty}\left|\lambda_{j}\right|^{q}||\left[b, \mu_{\Omega}\right]\left(b_{j}\right) \chi_{k}\right|_{L^{p \cdot(\cdot)}} ^{q / 2}\right) \\
& \times\left(\sum_{j=k}^{\infty}\left\|\left[b, \mu_{\Omega}\right]\left(b_{j}\right) \chi_{k}\right\|_{L^{p(\cdot)}}^{q^{\prime / 2}}\right)^{q / q^{\prime}} \\
& \leq \sup _{L \leq 0, L \in \mathbb{Z}} 2^{-L \lambda q} \sum_{k=0}^{L} 2^{k q \alpha_{\infty}}\left(\sum_{j=k}^{\infty}\left|\lambda_{j}\right|^{q}\left\|b_{j}\right\|_{L^{p(\cdot)}}^{q / 2}\right)\left(\sum_{j=k}^{\infty}\left\|b_{j}\right\|_{L^{p \cdot()}}^{q^{\prime} / 2}\right)^{q / q^{\prime}} \\
& \leq \sup _{L \leq 0, L \in \mathbb{Z}} 2^{-L \lambda q} \sum_{k=0}^{L} 2^{k q \alpha_{\infty}}\left(\sum_{j=k}^{\infty}\left|\lambda_{j}\right|^{q}\left|B_{j}\right|^{-\alpha_{j} q /(2 n)}\right)\left(\sum_{j=k}^{\infty}\left|B_{j}\right|^{-\alpha_{j} q^{\prime} /(2 n)}\right)^{q / q^{\prime}} \\
& \leq \sup _{L \leq 0, L \in \mathbb{Z}} 2^{-L \lambda q} \sum_{k=0}^{L} 2^{k q \alpha_{\infty}}\left(\sum_{j=k}^{\infty}\left|\lambda_{j}\right|^{q}\left|B_{j}\right|^{-\alpha_{j} q /(2 n)}\right) \\
& \leq \sup _{L \leq 0, L \in \mathbb{Z}} 2^{-L \lambda q} \sum_{k=0}^{L} 2^{\alpha_{\infty} k q / 2}\left(\sum_{j=k}^{\infty}\left|\lambda_{j}\right|^{q}\left|B_{j}\right|^{-\alpha_{j} q /(2 n)}\right) \\
& =\sup _{L \leq 0, L \in \mathbb{Z}} 2^{-L \lambda q} \sum_{j=0}^{L}\left|\lambda_{j}\right|^{q} \sum_{k=0}^{j} 2^{(k-j) \alpha_{\infty} q / 2}+\sup _{L \leq 0, L \in \mathbb{Z}} 2^{-L \lambda q} \sum_{j=L}^{\infty}\left|\lambda_{j}\right|^{q} \sum_{k=0}^{L} 2^{(k-j) \alpha_{\infty} q / 2} \\
& \leq \sup _{L \leq 0, L \in \mathbb{Z}} 2^{-L \lambda q} \sum_{j=0}^{L}\left|\lambda_{j}\right|^{q}+\sup _{L \leq 0, L \in \mathbb{Z}} \sum_{j=L}^{\infty} 2^{(j-L) \lambda q} 2^{-j \lambda q} \sum_{i=-\infty}^{j}\left|\lambda_{j}\right|^{q} \sum_{k=0}^{L} 2^{(k-j) \alpha_{\infty} q / 2} \\
& \leq \Delta+\Delta \sup _{L \leq 0, L \in \mathbb{Z}} \sum_{j=L}^{\infty} 2^{(j-L) \lambda q} 2^{(L-j) \alpha_{\infty} q / 2} \\
& \leq \Delta+\Delta \sup _{L \leq 0, L \in \mathbb{Z}} \sum_{j=L}^{\infty} 2^{(j-L) q\left(\alpha-\alpha_{\infty} / 2\right)} \\
& \leq \Delta .
\end{aligned}
$$

When $0<q \leq 1$, by $n \delta_{2} \leq \alpha(0), \alpha_{\infty}<\gamma+n \delta_{2}$ we get

$$
\begin{aligned}
I I I_{2}= & \sup _{L \leq 0, L \in \mathbb{Z}} 2^{-L \lambda q} \sum_{k=0}^{L} 2^{k q \alpha_{\infty}}\left(\sum_{j=\infty}^{k-1}\left|\lambda_{j}\right|\left\|\left[b, \mu_{\Omega}\right]\left(b_{j}\right) \chi_{k}\right\|_{L^{p(\cdot)}}\right)^{q} \\
\leq & \sup _{L \leq 0, L \in \mathbb{Z}} 2^{-L \lambda q} \sum_{k=0}^{L} 2^{k q \alpha_{\infty}}\left(C\|b\|_{L i p_{\gamma}}^{q} \sum_{j=\infty}^{k-1}\left|\lambda_{j}\right|^{q} 2^{\left[-j \alpha_{j}+(j-k)\left(\gamma+n \delta_{2}\right)\right] q}\right) \\
= & C\|b\|_{L i p_{\gamma}}^{q} \sup _{L \leq 0, L \in \mathbb{Z}} 2^{-L \lambda q} \sum_{k=0}^{L} 2^{k q \alpha_{\infty}}\left(\sum_{j=-\infty}^{-1}\left|\lambda_{j}\right|^{q} 2^{\left[-j \alpha(0)+(j-k)\left(\gamma+n \delta_{2}\right)\right] q}\right) \\
& +C\|b\|_{L i p_{\gamma}}^{q} \sup _{L \leq 0, L \in \mathbb{Z}} 2^{-L \lambda q} \sum_{k=0}^{L} 2^{k q \alpha(\infty)}\left(\sum_{j=0}^{k-1}\left|\lambda_{j}\right|^{q} 2^{\left[-j \alpha_{\infty}+(j-k)\left(\gamma+n \delta_{2}\right)\right] q}\right) \\
\leq & C\|b\|_{L i p_{\gamma}}^{q} \sup _{L \leq 0, L \in \mathbb{Z}} 2^{-L \lambda q} \sum_{k=0}^{L} 2^{k q\left[\alpha_{\infty}+\gamma+n \delta_{2}\right]} \sum_{j=-\infty}^{-1}\left|\lambda_{j}\right|^{q} 2^{\left[\gamma+n \delta_{2}+\alpha(0)\right] j q} \\
& +C\|b\|_{L i_{\gamma}}^{q} \sup _{L \leq 0, L \in \mathbb{Z}} 2^{-L \lambda q} \sum_{j=0}^{L}\left|\lambda_{j}\right|^{q} \sum_{k=j+1}^{\infty} 2^{\left[\gamma+n \delta_{2}-\alpha_{\infty}\right](j-k) q} \\
\leq & C\|b\|_{L i p_{\gamma}}^{q} \sup _{L \leq 0, L \in \mathbb{Z}} 2^{-L \lambda q} \sum_{j=-\infty}^{-1}\left|\lambda_{j}\right|^{q}+C\|b\|_{L i p_{\gamma}}^{q} \sup _{L \leq 0, L \in \mathbb{Z}} 2^{-L \lambda q} \sum_{j=0}^{L-1}\left|\lambda_{j}\right|^{q} \\
\leq & C\|b\|_{L i p_{\gamma}}^{q} \Delta .
\end{aligned}
$$


When $1<q<\infty$, let $1 / q+1 / q^{\prime}=1$. Since $n \delta_{2} \leq \alpha(0), \alpha_{\infty}<\gamma+n \delta_{2}$, and by Hölder's inequality, we have

$$
\begin{aligned}
& I I I_{2}=\sup _{L \leq 0, L \in \mathbb{Z}} 2^{-L \lambda q} \sum_{k=0}^{L} 2^{k q \alpha(\infty)}\left(\sum_{j=-\infty}^{k-1}\left|\lambda_{j}\right|\left\|\left[b, \mu_{\Omega}\right]\left(b_{j}\right) \chi_{k}\right\|_{L^{p(\cdot)}}\right)^{q} \\
& \leq \sup _{L \leq 0, L \in \mathbb{Z}} 2^{-L \lambda q} \sum_{k=0}^{L} 2^{k q \alpha(\infty)}\left(\sum_{j=-\infty}^{-1} C\|b\|_{L i p_{\gamma}}^{q}\left|\lambda_{j}\right| 2^{\left[-j \alpha_{j}+(j-k)\left(\gamma+n \delta_{2}\right)\right]}\right)^{q} \\
& \leq C\|b\|_{L i p_{\gamma}}^{q} \sup _{L \leq 0, L \in \mathbb{Z}} 2^{-L \lambda q} \sum_{k=0}^{L} 2^{k q \alpha(\infty)}\left(\sum_{j=-\infty}^{-1}\left|\lambda_{j}\right| 2^{\left[-j \alpha(0)+(j-k)\left(\gamma+n \delta_{2}\right)\right]}\right)^{q} \\
& +C\|b\|_{L i p_{\gamma}}^{q} \sup _{L \leq 0, L \in \mathbb{Z}} 2^{-L \lambda q} \sum_{k=0}^{L} 2^{k q \alpha(\infty)}\left(\sum_{j=0}^{k-1}\left|\lambda_{j}\right| 2^{\left[-j \alpha_{\infty}+(j-k)\left(\gamma+n \delta_{2}\right)\right]}\right)^{q} \\
& \leq C\|b\|_{L i p_{\gamma}}^{q} \sup _{L \leq 0, L \in \mathbb{Z}} 2^{-L \lambda q} \sum_{k=0}^{L} 2^{k q\left[\alpha_{\infty}-\left(\gamma+n \delta_{2}\right)\right]}\left(\sum_{j=-\infty}^{-1}\left|\lambda_{j}\right| 2^{\left[\left(\gamma+n \delta_{2}\right)-\alpha(0)\right] j}\right)^{q} \\
& +C\|b\|_{L i p_{\gamma}}^{q} \sup _{L \leq 0, L \in \mathbb{Z}} 2^{-L \lambda q} \sum_{k=0}^{L}\left(\sum_{j=0}^{k-1}\left|\lambda_{j}\right| 2^{(j-k)\left[\gamma+n \delta_{2}-\alpha_{\infty}\right]}\right)^{q} \\
& \leq\left(C\|b\|_{L i p_{\gamma}}^{q} \sup _{L \leq 0, L \in \mathbb{Z}} 2^{-L \lambda q} \sum_{j=-\infty}^{-1}\left|\lambda_{j}\right|^{q} 2^{\left[\left(\gamma+n \delta_{2}\right)-\alpha(0)\right] j g / 2}\right) \\
& \times\left(\sum_{j=-\infty}^{-1}\left|\lambda_{j}\right|^{q} 2^{\left[\left(\gamma+n \delta_{2}\right)-\alpha(0)\right] j q^{\prime} / 2}\right)^{q / q^{\prime}} \\
& +C\|b\|_{L i p_{\gamma}}^{q} \sup _{L \leq 0, L \in \mathbb{Z}} 2^{-L \lambda q} \sum_{k=0}^{L}\left(\sum_{j=0}^{k-1}\left|\lambda_{j}\right|^{q} 2^{(j-k)\left[\gamma+n \delta_{2}-\alpha_{\infty}\right] q / 2}\right) \\
& \times\left(\sum_{j=0}^{k-1}\left|\lambda_{j}\right| 2^{(j-k)\left[\gamma+n \delta_{2}-\alpha_{\infty}\right] q^{\prime} / 2}\right)^{q / q^{\prime}} \\
& \leq C\|b\|_{L i p_{\gamma}}^{q} \sup _{L \leq 0, L \in \mathbb{Z}} 2^{-L \lambda q} \sum_{j=-\infty}^{-1}\left|\lambda_{j}\right|^{q} 2^{\left[\left(\gamma+n \delta_{2}\right)-\alpha(0)\right] j q / 2} \\
& \left.+C\|b\|_{L i p_{\gamma}}^{q} \sup _{L \leq 0, L \in \mathbb{Z}} 2^{-L \lambda q} \sum_{k=0}^{L} \sum_{j=0}^{k-1}\left|\lambda_{j}\right|^{q} 2^{(j-k)\left[\gamma+n \delta_{2}-\alpha_{\infty}\right]}\right] q / 2 \\
& \leq C\|b\|_{L i p_{\gamma}}^{q} \sup _{L \leq 0, L \in \mathbb{Z}} 2^{-L \lambda q} \sum_{j=-\infty}^{-1}\left|\lambda_{j}\right|^{q} \\
& +C\|b\|_{L i p_{\gamma}}^{q} \sup _{L \leq 0, L \in \mathbb{Z}} 2^{-L \lambda q} \sum_{j=0}^{L-1}\left|\lambda_{j}\right|^{q} \sum_{k=j+1}^{L} 2^{(j-k)\left[\gamma+n \delta_{2}-\alpha_{\infty}\right] q / 2} \\
& \leq C\|b\|_{L i p_{\gamma}}^{q} \sup _{L \leq 0, L \in \mathbb{Z}} 2^{-L \lambda q} \sum_{j=-\infty}^{-1}\left|\lambda_{j}\right|^{q}+C\|b\|_{L i p_{\gamma}}^{q} \sup _{L \leq 0, L \in \mathbb{Z}} 2^{-L \lambda q} \sum_{j=0}^{L-1}\left|\lambda_{j}\right|^{q} \\
& \leq C\|b\|_{L i p_{y}}^{q} \Delta .
\end{aligned}
$$

Joint the estimates for I, II and III, we obtain

$$
\left\|\left[b, \mu_{\Omega}\right](f)\right\|_{M K_{p(), \lambda}^{\alpha(\cdot), q}\left(\mathbb{R}^{n}\right)}^{q} \leq C\|b\|_{L p_{\gamma}}^{q}\|f\|_{H M K_{p((), \lambda}^{\alpha(\cdot), q}} .
$$

Then we complete the proof of Theorem 3.1.

\section{BMO Boundedness for the Commutator of Marcikiewicz Integrals Operator}

In this section, we prove the boundedness of the commutator of Marcikiewicz 
integrals on Herz-Morrey-Hrdy spaces with variable exponent with function $b \in B M O\left(\mathbb{R}^{n}\right)$.

Theorem 4.1.

Suppose that $b \in B M O\left(\mathbb{R}^{n}\right)$ with $0<\gamma \leq 1$. If $p(\cdot) \in \mathcal{P}\left(\mathbb{R}^{n}\right)$ satisfies proposition 2.3 and $\Omega \in L^{s}\left(S^{n-1}\right)\left(s>q^{\prime-}\right)$. Let $0<p_{1} \leq p_{2}<\infty$ and $0<\lambda<\alpha<n \delta_{2}-\gamma-\frac{n}{s} \quad\left(\right.$ or $\left.\quad 0<\lambda<\alpha_{1} \leq \alpha_{1}<n \delta_{2}-\gamma-\frac{n}{s}\right)$. Then $\left[b, \mu_{\Omega}\right]$ is bounded from $H M \dot{K}_{p(\cdot), \lambda}^{\alpha(\cdot), q}\left(\mathbb{R}^{n}\right) \quad\left(\right.$ or $\left.H M K_{p(\cdot), \lambda}^{\alpha(\cdot), q}\left(\mathbb{R}^{n}\right)\right)$ to $M \dot{K}_{p(\cdot), \lambda}^{\alpha(\cdot), q}\left(\mathbb{R}^{n}\right)$ (or $\left.M K_{p(\cdot), \lambda}^{\alpha(\cdot, q}\left(\mathbb{R}^{n}\right)\right)$.

\section{proof:}

In a way similar to theorem (3.2) we only prove the homogeneous case. Let $b \in B M O\left(\mathbb{R}^{n}\right)$ and $f \in H M K_{p(\cdot), \lambda}^{\alpha(\cdot), q}\left(\mathbb{R}^{n}\right)$. Let us write

$$
f(x)=\sum_{j=0}^{\infty} f(x) \chi_{j}(x)=\sum_{j=0}^{\infty} f_{j}(x) \text {. }
$$

Then we have

$$
\begin{gathered}
\left\|\left[b, \mu_{\Omega}\right](f)\right\|_{M K_{p(\cdot), \lambda}^{\alpha(\cdot), q}\left(\mathbb{R}^{n}\right)=}^{q} \max \left\{\sup _{L \leq 0, L \in \mathbb{Z}} 2^{-L \lambda q} \sum_{k=-\infty}^{L} 2^{k q \alpha(0)}\left\|\left[b, \mu_{\Omega}\right](f) \chi_{k}\right\|_{L^{p(\cdot)}}^{q},\right. \\
\quad \sup _{L \leq 0, L \in Z} 2^{-L \lambda q}\left(\sum_{k=-\infty}^{-1} 2^{k q \alpha(0)}\left\|\left[b, \mu_{\Omega}\right](f) \chi_{k}\right\|_{L^{p(\cdot)}}^{q}\right. \\
\left.\left.\quad+\sum_{k=0}^{L} 2^{k q \alpha(\infty)}\left\|\left[b, \mu_{\Omega}\right](f) \chi_{k}\right\|_{L^{p(\cdot)}}^{q}\right)\right\} \\
:=\max \{H, H H+H H H\} . \\
H=\sup _{L \leq 0, L \in \mathbb{Z}} 2^{-L \lambda q} \sum_{k=-\infty}^{L} 2^{k q \alpha(0)}\left\|\left[b, \mu_{\Omega}\right](f) \chi_{k}\right\|_{L^{p(\cdot)}}^{q}, \\
H H=\sum_{k=-\infty}^{-1} 2^{k q \alpha(0)}\left\|\left[b, \mu_{\Omega}\right](f) \chi_{k}\right\|_{L^{p(\cdot)}}^{q}, \\
H H H=\sup _{L \leq 0, L \in \mathbb{Z}} 2^{-L \lambda q} \sum_{k=0}^{L} 2^{k q \alpha(\infty)}\left\|\left[b, \mu_{\Omega}\right](f) \chi_{k}\right\|_{L^{p(\cdot)}}^{q} .
\end{gathered}
$$

From the Hölder's inequality, we have

$$
\begin{aligned}
& \left|\left[b, \mu_{\Omega}\right]\left(b_{j}\right) \chi_{k}\right|_{L^{p(\cdot)}\left(\mathbb{R}^{n}\right)} \\
& \leq C \int_{B_{j}} \frac{|\Omega(x-y)|}{|x-y|^{n}}|b(x)-b(y)|\left|f_{j}(y)\right| \mathrm{d} y \\
& \leq C 2^{-k n} \int_{B_{j}}|\Omega(x-y)||b(x)-b(y)|\left|f_{j}(y)\right| \mathrm{d} y \\
& \leq C 2^{-k n}\left(\left|b(x)-b_{B_{j}}\right| \int_{B_{j}}|\Omega(x-y)|\left|f_{j}(y)\right| \mathrm{d} y\right. \\
& \left.\quad+\int_{B_{j}}|\Omega(x-y)|\left|b_{B_{j}}-b(y)\right|\left|f_{j}(y)\right| \mathrm{d} y\right) \\
& \leq C 2^{-k n}\left(\left|b(x)-b_{B_{j}}\right|\left\|\Omega(x-\cdot) \chi_{j}(\cdot)\right\|_{L^{q^{\prime} \cdot(\cdot)}\left(\mathbb{R}^{n}\right)}\left\|f_{j}\right\|_{L^{q(\cdot)}\left(\mathbb{R}^{n}\right)} \mathrm{d} y\right. \\
& \left.\quad+\left\|\Omega(x-\cdot)\left(b_{B_{j}}-b(\cdot)\right) \chi_{j}(\cdot)\right\|_{L^{q^{\prime} \cdot(\cdot)}\left(\mathbb{R}^{n}\right)}\left\|f_{j}\right\|_{L^{q(\cdot)}\left(\mathbb{R}^{n}\right)} \mathrm{d} y\right) .
\end{aligned}
$$


O. Khalil et al.

Noting $s>q^{\prime-}$, we denote $\tilde{q}^{\prime}(\cdot)>1$ and $\frac{1}{q^{\prime}(x)}=\frac{1}{\tilde{q}^{\prime}(x)}+\frac{1}{s}$. By lemmas 3.2, 3.10 we have

$$
\begin{aligned}
& \left\|\Omega(x-\cdot) \chi_{j}(\cdot)\right\|_{L^{\left.q^{(} \cdot()\right)}\left(\mathbb{R}^{n}\right)} \leq\left\|\Omega(x-\cdot) \chi_{j}(\cdot)\right\|_{L^{S^{(\cdot)}}\left(\mathbb{R}^{n}\right)}\left\|\chi_{j}(\cdot)\right\|_{L^{\tilde{q}^{2}(\cdot)}\left(\mathbb{R}^{n}\right)} \\
& \leq\left\|\Omega(x-\cdot) \chi_{j}(\cdot)\right\|_{L^{(\cdot)}\left(\mathbb{R}^{n}\right)}\left\|\chi_{B_{j}}(\cdot)\right\|_{L^{q_{2}(\cdot)}\left(\mathbb{R}^{n}\right)} \\
& \leq 2^{-j \gamma}\left(\int_{A_{j}}|y|^{s \gamma}|\Omega(x-y)|^{s} \mathrm{dy}\right)^{\frac{1}{s}}\left\|\chi_{B_{j}}(\cdot)\right\|_{L^{\tilde{q}_{2}(\cdot)}\left(\mathbb{R}^{n}\right)} \\
& \leq C 2^{-j \gamma}|2|^{k\left(\gamma+\frac{n}{s}\right)}\|\Omega\|_{L^{s}\left(s^{n-1}\right)}\left\|\chi_{B_{j}}(\cdot)\right\|_{L^{\hat{q}^{\prime}}(\cdot)\left(\mathbb{R}^{n}\right)} \cdot
\end{aligned}
$$

By lemma (2.12), when $\left|B_{j}\right| \leq 2^{n}, x_{j} \in B_{j}$ and when $\left|B_{k}\right| \geq 1$ respectively we have

$$
\left\|\chi_{B_{j}}\right\|_{L^{\tilde{q}^{(}(\cdot)\left(\mathbb{R}^{n}\right)}} \approx\left|B_{k}\right| \frac{1}{\tilde{q}^{1}\left(\chi_{k}\right)} \approx\left\|\chi_{B_{j}}\right\|_{L^{q^{\prime}(\cdot)}\left(\mathbb{R}^{n}\right)}\left|B_{j}\right|^{\frac{-1}{s}}
$$

and

$$
\left\|\chi_{B_{j}}\right\|_{L^{\tilde{q}^{\prime}(\cdot)\left(\mathbb{R}^{n}\right)}} \approx\left|B_{j}\right|^{\frac{1}{\tilde{q}^{\prime}(\infty)}} \approx\left\|\chi_{B_{j}}\right\|_{L^{q^{\prime}(\cdot)}\left(\mathbb{R}^{n}\right)}\left|B_{j}\right|^{\frac{-1}{s}},
$$

we obtain $\left\|\chi_{B_{j}}\right\|_{L_{q^{\prime}()()\left(\mathbb{R}^{n}\right)}} \approx\left\|\chi_{B_{j}}\right\|_{L^{q^{\prime}()(}\left(\mathbb{R}^{n}\right)}\left|B_{j}\right|^{\frac{-1}{s}}$.
$\quad$ So we have

$$
\left\|\Omega(x-\cdot) \chi_{j}(\cdot)\right\|_{L^{q^{\prime}(\cdot)\left(\mathbb{R}^{n}\right)}} \leq C 2^{(k-j)\left(\gamma+\frac{n}{s}\right)}\|\Omega\|_{L^{s}\left(S^{n-1}\right)}\left\|\chi_{B_{j}}(\cdot)\right\|_{L^{q^{(}(\cdot)}\left(\mathbb{R}^{n}\right)} .
$$

Similarly by lemma 2.4 we have

$$
\begin{aligned}
& \left\|\Omega(x-\cdot)\left(b_{B_{j}}-b(\cdot)\right) \chi_{j}(\cdot)\right\|_{L^{q^{\prime} \cdot(\cdot)\left(\mathbb{R}^{n}\right)}} \\
& \leq\left\|\Omega(x-\cdot) \chi_{j}(\cdot)\right\|_{L^{s}\left(\mathbb{R}^{n}\right)}\left\|\left(b_{B_{j}}-b(\cdot)\right) \chi_{j}(\cdot)\right\|_{L^{q^{(}()}\left(\mathbb{R}^{n}\right)} \\
& \leq C\|b\|_{*}\left\|\chi_{B_{j}}(\cdot)\right\|_{L^{\tilde{q}^{2}(\cdot)}\left(\mathbb{R}^{n}\right)}\left\|\Omega(x-\cdot) \chi_{j}(\cdot)\right\|_{L^{s}\left(\mathbb{R}^{n}\right)} \\
& \leq C\|b\|_{*} 2^{(k-j)\left(\gamma+\frac{n}{s}\right)}\|\Omega\|_{L^{s}\left(s^{n-1}\right)}\left\|\chi_{B_{j}}\right\|_{L^{q^{(} \cdot()}\left(\mathbb{R}^{n}\right)} .
\end{aligned}
$$

Now, by (4.1), (4.2), lemmas 2.4, 2.5 and 2.3, we have

$$
\begin{aligned}
& \left\|\left[b, \mu_{\Omega}\right]\left(f_{j}\right) \chi_{k}\right\|_{L^{q(\cdot)}\left(\mathbb{R}^{n}\right)} \\
& \leq C 2^{-k n}\left(2^{(k-j)\left(\gamma+\frac{n}{s}\right)}\|\Omega\|_{L^{s}\left(s^{n-1}\right)}\left\|\chi_{B_{j}}\right\|_{L^{q^{(\cdot)}}\left(\mathbb{R}^{n}\right)}\left\|\left(b(\cdot)-b_{B_{j}}\right) \chi_{k}(\cdot)\right\|_{L^{q^{(\cdot)}\left(\mathbb{R}^{n}\right)}}\left\|f_{j}\right\|_{L^{q(\cdot)}\left(\mathbb{R}^{n}\right)}\right. \\
& \left.+\|b\|_{*} 2^{(k-j)\left(\gamma+\frac{n}{s}\right)}\|\Omega\|_{L^{s}\left(S^{n-1}\right)}\left\|\chi_{B_{j}}\right\|_{L^{q^{(}(\cdot)}\left(\mathbb{R}^{n}\right)}\left\|\chi_{k}\right\|_{L^{q(\cdot)}\left(\mathbb{R}^{n}\right)}\left\|f_{j}\right\|_{L^{(\cdot)}\left(\mathbb{R}^{n}\right)} \mathrm{d} y\right) \\
& \leq C 2^{-k n}\left((k-j)\|b\|_{*} 2^{(k-j)\left(\gamma+\frac{n}{s}\right)}\|\Omega\|_{L^{s}\left(s^{n-1}\right)}\left\|\chi_{B_{j}}\right\|_{L^{q}(\cdot)\left(\mathbb{R}^{n}\right)}\left\|\chi_{B_{k}}\right\|_{L^{(\cdot)}\left(\mathbb{R}^{n}\right)}\left\|f_{j}\right\|_{L^{q(\cdot)}\left(\mathbb{R}^{n}\right)}\right.
\end{aligned}
$$

DOI: 10.4236/am.2021.125030

438

Applied Mathematics 


$$
\begin{aligned}
& \left.+\|b\|_{*} 2^{(k-j)\left(\gamma+\frac{n}{s}\right)}\|\Omega\|_{L^{s}\left(s^{n-1}\right)}\left\|\chi_{B_{j}}\right\|_{L^{q^{(} \cdot()}\left(\mathbb{R}^{n}\right)}\left\|\chi_{B_{k}}\right\|_{L^{q(\cdot)}\left(\mathbb{R}^{n}\right)}\left\|f_{j}\right\|_{L^{q(\cdot)}\left(\mathbb{R}^{n}\right)} \mathrm{d} y\right) \\
& \leq C(k-j)\|b\|_{*} 2^{-k n} 2^{(k-j)\left(\gamma+\frac{n}{s}\right)}\|\Omega\|_{L^{(}\left(S^{n-1}\right)}\left\|\chi_{B_{j}}\right\|_{L^{q}(\cdot)\left(\mathbb{R}^{n}\right)}\left\|\chi_{B_{k}}\right\|_{L^{q^{i}(\cdot)}\left(\mathbb{R}^{n}\right)}\left\|f_{j}\right\|_{L^{q(\cdot)}\left(\mathbb{R}^{n}\right)}
\end{aligned}
$$

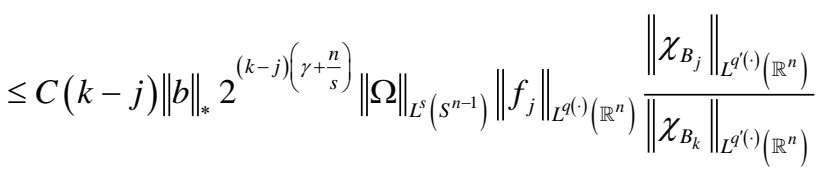

$$
\begin{aligned}
& \leq C\|b\|_{*}(k-j) 2^{(k-j)\left(n \delta_{2}-\gamma-\frac{n}{s}\right)}\|\Omega\|_{L^{s}\left(s^{n-1}\right)}\left\|f_{j}\right\|_{L^{q^{(.)}}\left(\mathbb{R}^{n}\right)} .
\end{aligned}
$$

By the boundedness of $\mu_{\Omega}$ in $L^{p(\cdot)}$ see [7], we have

$$
\left\|\left(\mu_{\Omega} f_{j}\right) \chi_{k}\right\|_{L^{p(\cdot)}} \leq\left\|f_{j}\right\|_{L^{p(\cdot)}} \leq\left|B_{j}\right|^{-\alpha_{j} / n}=2^{-j \alpha_{j}} .
$$

So we have

$$
\left\|\left[b, \mu_{\Omega}\right]\left(f_{j}\right) \chi_{k}\right\|_{L^{(\cdot)}\left(\mathbb{R}^{n}\right)} \leq\|b\|_{*}(k-j)\|\Omega\|_{L^{s}\left(S^{n-1}\right)} 2^{(k-j)\left(n \delta_{2}-\gamma-\frac{n}{s}\right)-j \alpha_{j}} .
$$

Firstly we estimate $H$. We need to show that there exists a positive constant $C$, such that $H \leq C \Delta$ Consider

$$
\begin{aligned}
H= & \sup _{L \leq 0, L \in \mathbb{Z}} 2^{-L \lambda q} \sum_{k=-\infty}^{L} 2^{k q \alpha(0)}\left\|\left[b, \mu_{\Omega}\right](f) \chi_{k}\right\|_{L^{p(\cdot)}}^{q} \\
\leq & \sup _{L \leq 0, L \in \mathbb{Z}} 2^{-L \lambda q q} \sum_{k=-\infty}^{L} 2^{k q \alpha(0)}\left(\sum_{j=k}^{\infty}\left|\lambda_{j}\right|\left\|\left[b, \mu_{\Omega}\right]\left(f_{j}\right) \chi_{k}\right\|_{L^{p(\cdot)}}^{q}\right)^{q} \\
& +\sup _{L \leq 0, L \in \mathbb{Z}} 2^{-L \lambda q} \sum_{k=-\infty}^{L} 2^{k \alpha \alpha(0)}\left(\sum_{j=-\infty}^{k-1}\left|\lambda_{j}\right| \|\left.\left[b, \mu_{\Omega}\right]\left(f_{j}\right) \chi_{k}\right|_{L^{p(\cdot)}} ^{q}\right)^{q} \\
& :=H_{1}+H_{2} .
\end{aligned}
$$

By boundedness of $\left[b, \mu_{\Omega}\right]$ in $L^{p(\cdot)}$, see $([20])$, when $0<q \leq 1$ we have

$$
\begin{aligned}
H_{1}= & \sup _{L \leq 0, L \in \mathbb{Z}} 2^{-L \lambda q} \sum_{k=-\infty}^{L} 2^{k q \alpha(0)}\left(\left.\sum_{j=k}^{\infty}\left|\lambda_{j}\right|||\left[b, \mu_{\Omega}\right]\left(f_{j}\right) \chi_{k}\right|_{L^{p(\cdot)}} ^{q}\right)^{q} \\
\leq & \sup _{L \leq 0, L \in \mathbb{Z}} 2^{-L \lambda q} \sum_{k=-\infty}^{L} 2^{k q \alpha(0)}\left(\sum_{j=k}^{\infty}\left|\lambda_{j}\right| 2^{-j \alpha_{j}}\right)^{q} \\
\leq & \sup _{L \leq 0, L \in \mathbb{Z}} 2^{-L \lambda q} \sum_{k=-\infty}^{L} 2^{k q \alpha(0)}\left(\sum_{j=k}^{-1}\left|\lambda_{j}\right| 2^{-j \alpha(0) j q}+\sum_{j=0}^{\infty}\left|\lambda_{j}\right| 2^{-j \alpha_{\infty} q}\right) \\
\leq & \sup _{L \leq 0, L \in \mathbb{Z}} 2^{-L \lambda q} \sum_{k=-\infty}^{L} \sum_{j=k}^{-1}\left|\lambda_{j}\right|^{q} 2^{\alpha(0)(k-j) q} \\
& +\sup _{L \leq 0, L \in \mathbb{Z}} 2^{-L \lambda q} \sum_{k=-\infty}^{L} 2^{k q \alpha(0)} \sum_{j=0}^{\infty}\left|\lambda_{j}\right|^{q} 2^{-j \alpha_{\infty} q} \\
\leq & \sup _{L \leq 0, L \in \mathbb{Z}} 2^{-L \lambda q} \sum_{j=-\infty}^{-1}\left|\lambda_{j}\right|^{q} \sum_{k=-\infty}^{j} 2^{\alpha(0)(k-j) q} \\
& +\sup _{L \leq 0, L \in \mathbb{Z} j=0} \sum^{\infty} 2^{-j \lambda q}\left|\lambda_{j}\right|^{q} 2^{\left(\lambda-\alpha_{\infty}\right) j q} 2^{-L \lambda q} \sum_{k=-\infty}^{L} 2^{k q \alpha(0)}
\end{aligned}
$$




$$
\begin{aligned}
& \leq \sup _{L \leq 0, L \in \mathbb{Z}} 2^{-L \lambda q} \sum_{j=-\infty}^{L}\left|\lambda_{j}\right|^{q}+\sup _{L \leq 0, L \in \mathbb{Z}} 2^{-L \lambda q} \sum_{j=L}^{-1}\left|\lambda_{j}\right|^{q} \sum_{k=-\infty}^{j} 2^{\alpha(0)(k-j) q} \\
& +\Delta \sup _{L \leq 0, L \in \mathbb{Z} j=0} \sum^{\infty} 2^{\left(\lambda-\alpha_{\infty}\right) j q} \sum_{k=-\infty}^{L} 2^{(\alpha(0) k-L \lambda) q} \\
& \leq \Delta+\sup _{L \leq 0, L \in \mathbb{Z} j=L} \sum^{-1} 2^{-j \lambda q}\left|\lambda_{j}\right|^{q} 2^{(j-L) \lambda q} \sum_{k=-\infty}^{j} 2^{\alpha(0)(k-j) q}+\Delta \\
& \leq \Delta+\sup _{L \leq 0, L \in \mathbb{Z}_{j=L}} 2^{-j \lambda q}\left|\lambda_{j}\right|^{q} 2^{(j-L) \lambda q} \sum_{k=-\infty}^{j} 2^{\alpha(0)(k-j) q} \\
& \leq \Delta .
\end{aligned}
$$

When $1<q<\infty$ and $1 / q+1 / q^{\prime}=1$, and let $\gamma+n \delta_{2}-\alpha>0$, we have

$$
\begin{aligned}
& H_{1}=\sup _{L \leq 0, L \in \mathbb{Z}} 2^{-L \lambda q} \sum_{k=-\infty}^{L} 2^{k q \alpha(0)}\left(\sum_{j=k}^{\infty}\left|\lambda_{j}\right|||\left[b, \mu_{\Omega}\right]\left(f_{j}\right) \chi_{k} \|_{L^{p(\cdot)}}^{q}\right)^{q} \\
& \leq \sup _{L \leq 0, L \in \mathbb{Z}} 2^{-L \lambda q} \sum_{k=-\infty}^{L} 2^{k q \alpha(0)}\left(\sum_{j=k}^{\infty}\left|\lambda_{j}\right| 2^{-j \alpha}\right)^{q} \\
& \leq \sup _{L \leq 0, L \in \mathbb{Z}} 2^{-L \lambda q} \sum_{k=-\infty}^{L}\left(\sum_{j=k}^{-1}\left|\lambda_{j}\right| 2^{\alpha(0)(k-j)}\right)^{q} \\
& +\sup _{L \leq 0, L \in \mathbb{Z}} 2^{-L \lambda q} \sum_{k=-\infty}^{L} 2^{k q \alpha(0)}\left(\sum_{j=0}^{\infty}\left|\lambda_{j}\right| 2^{-j \alpha_{\infty}}\right)^{q} \\
& \leq \sup _{L \leq 0, L \in \mathbb{Z}} 2^{-L \lambda q} \sum_{k=-\infty}^{L}\left(\sum_{j=k}^{-1}\left|\lambda_{j}\right|^{q} 2^{\alpha(0)(k-j) q / 2}\right)\left(\sum_{j=k}^{-1} 2^{\alpha(0)(k-j) q^{\prime} / 2}\right)^{q / q^{\prime}} \\
& +\sup _{L \leq 0, L \in \mathbb{Z}} 2^{-L \lambda q} \sum_{k=-\infty}^{L} 2^{k q \alpha(0)}\left(\sum_{j=0}^{\infty}\left|\lambda_{j}\right|^{q} 2^{-j \alpha_{\infty} q / 2}\right)\left(\sum_{j=0}^{\infty} 2^{-j \alpha_{\infty} q^{\prime} / 2}\right)^{q / q^{\prime}} \\
& \leq \sup _{L \leq 0, L \in \mathbb{Z}} 2^{-L \lambda q} \sum_{k=-\infty}^{L} \sum_{j=k}^{-1}\left|\lambda_{j}\right|^{q} 2^{(j-k) q / 2} \\
& +\sup _{L \leq 0, L \in \mathbb{Z}} 2^{-L \lambda q} \sum_{k=-\infty}^{L} 2^{k q \alpha(0)} \sum_{j=0}^{\infty}\left|\lambda_{j}\right|^{q} 2^{-j \alpha_{\infty} q / 2} \\
& \leq \sup _{L \leq 0, L \in \mathbb{Z}} 2^{-L \lambda q} \sum_{j=-\infty}^{-1}\left|\lambda_{j}\right|^{q} \sum_{k=-\infty}^{j} 2^{\alpha(0)(j-k) q / 2} \\
& +\sup _{L \leq 0, L \in \mathbb{Z}} \sum_{j=0}^{\infty} 2^{j \lambda q}\left|\lambda_{j}\right|^{q} 2^{\left(\lambda-\alpha_{\infty} / 2\right) j q} 2^{-L \lambda q} \sum_{k=-\infty}^{L} 2^{k q \alpha(0)} \\
& \leq \sup _{L \leq 0, L \in \mathbb{Z}} 2^{-L \lambda q} \sum_{j=-\infty}^{L}\left|\lambda_{j}\right|^{q}+\sup _{L \leq 0, L \in \mathbb{Z}} 2^{-L \lambda q} \sum_{j=L}^{-1}\left|\lambda_{j}\right|^{q} \sum_{j=-\infty}^{j} 2^{\alpha(0)(k-j) q / 2} \\
& +\Delta \sup _{L \leq 0, L \in \mathbb{Z}} \sum_{j=0}^{\infty} 2^{\left(\lambda-\alpha_{\infty} / 2\right) j / 2 q} \sum_{k=-\infty}^{L} 2^{k q \alpha(0)-L \lambda q} \\
& \leq \Delta+\sup _{L \leq 0, L \in \mathbb{Z}} \sum_{j=L}^{-1} 2^{-j \lambda q}\left|\lambda_{j}\right|^{q} 2^{(j-L) \lambda q} \sum_{j=-\infty}^{j} 2^{\alpha(0)(k-j) q / 2}+\Delta \\
& \leq \Delta+\Delta \sup _{L \leq 0, L \in \mathbb{Z}} \sum_{j=L}^{-1} 2^{(j-L) \lambda q} \sum_{j=-\infty}^{j} 2^{\alpha(0)(k-j) q / 2}
\end{aligned}
$$

$\leq \Delta$

Now we estimate $H_{2}$, when $0<q \leq 1$, by $n \delta_{2} \leq \alpha(0)<n \delta_{2}-\gamma-\frac{n}{s}$, we get 


$$
\begin{aligned}
& H_{2}=\sup _{L \leq 0, L \in \mathbb{Z}} 2^{-L \lambda q} \sum_{k=-\infty}^{L} 2^{k q \alpha(0)}\left(\sum_{j=-\infty}^{k-1}\left|\lambda_{j}\right|\left\|\left[b, \mu_{\Omega}\right]\left(f_{j}\right) \chi_{k}\right\|_{L^{p}(\cdot)}^{q}\right)^{q} \\
& \leq C\|b\|_{*}^{q}\|\Omega\|_{L^{s}\left(S^{n-1}\right)}^{q} \sup _{L \leq 0, L \in \mathbb{Z}} 2^{-L \lambda q} \sum_{k=-\infty}^{L} 2^{k q \alpha(0)}\left(\sum_{j=-\infty}^{k-1}\left|\lambda_{j}\right|^{q}(k-j)^{q} 2^{\left[-j \alpha(0)+(j-k)\left(n \delta_{2}-\gamma-\frac{n}{s}\right)\right] q}\right) \\
& \leq C\|b\|_{*}^{q}\|\Omega\|_{L^{s}\left(S^{n-1}\right)}^{q} \sup _{L \leq 0, L \in \mathbb{Z}} 2^{-L \lambda q} \sum_{k=-\infty}^{L}\left|\lambda_{j}\right|^{q} \sum_{j=k}^{\infty}(k-j)^{q} 2^{q(j-k)\left(n \delta_{2}-\gamma-\frac{n}{s}-\alpha(0)\right)} \\
& \leq C\|b\|_{*}^{q}\|\Omega\|_{L^{s}\left(s^{n-1}\right)}^{q} \Delta .
\end{aligned}
$$

when $1<q<\infty$, let $1 / q+1 / q^{\prime}=1$. Since $n \delta_{2}<\alpha(0) \leq \gamma+n \delta_{2}$, by Hölder's inequality we have

$$
\begin{aligned}
& H_{2}=\sup _{L \leq 0, L \in \mathbb{Z}} 2^{-L \lambda q} \sum_{k=-\infty}^{L} 2^{k q \alpha(0)}\left(\sum_{j=-\infty}^{k-1}\left|\lambda_{j}\right|\left\|\left[b, \mu_{\Omega}\right]\left(f_{j}\right) \chi_{k}\right\|_{L^{p(\cdot)}}^{q}\right)^{q} \\
& \leq C\|b\|_{*}^{q}\|\Omega\|_{L^{s}\left(s^{n-1}\right)}^{q} \sup _{L \leq 0, L \in \mathbb{Z}} 2^{-L \lambda q} \sum_{k=-\infty}^{L} 2^{k q \alpha(0)}\left(\sum_{j=-\infty}^{k-1}\left|\lambda_{j}\right|(k-j) 2^{\left[-j \alpha+(j-k)\left(n \delta_{2}-\gamma-\frac{n}{s}\right)\right]}\right)^{q} \\
& \leq C\|b\|_{*}^{q}\|\Omega\|_{L^{s}\left(n^{n-1}\right)}^{q} \sup _{L \leq 0, L \in \mathbb{Z}} 2^{-L \lambda q} \sum_{k=-\infty}^{L} 2^{k q \alpha(0)}\left(\sum_{j=k}^{k-1}\left|\lambda_{j}\right|^{q}(k-j)^{q} 2^{\left[-j \alpha+(j-k)\left(n \delta_{2}-\gamma-\frac{n}{s}\right)\right] q / 2}\right) \\
& \times\left(\sum_{j=0}^{\infty} 2^{\left[-j \alpha+(j-k)\left(n \delta_{2}-\gamma-\frac{n}{s}\right)\right] q / 2}\right)^{q / q^{\prime}} \\
& \left.\left.\leq C\|b\|_{*}^{q}\|\Omega\|_{L^{s}\left(s^{n-1}\right)}^{q} \sup _{L \leq 0, L \in \mathbb{Z}} 2^{-L \lambda q} \sum_{k=-\infty}^{L} 2^{k q \alpha(0)}\left(\sum_{j=k}^{k-1}\left|\lambda_{j}\right|^{q}(k-j)^{q} 2^{\left[-j \alpha+(j-k)\left(n \delta_{2}-\gamma-\frac{n}{s}\right)\right.}\right]\right]^{\alpha / 2}\right) \\
& \left.\leq C\|b\|_{*}^{q}\|\Omega\|_{L^{s}}^{q}\left(S^{n-1}\right) \sup _{L \leq 0, L \in \mathbb{Z}} 2^{-L \lambda q} \sum_{k=-\infty}^{L}\left|\lambda_{j}\right|^{q} \sum_{k=j+1}^{-1}(k-j)^{q} 2^{\left[+(j-k)\left(n \delta_{2}-\gamma-\frac{n}{s}-\alpha\right)\right]}\right] q / 2 \\
& \leq C\|b\|_{*}^{q}\|\Omega\|_{L^{s}\left(s^{n-1}\right)}^{q} \Delta .
\end{aligned}
$$

Secondly, we estimate $H H$. We need to show that there exists a positive constant $C$, such that $H H \leq C \Delta$ Consider

$$
\begin{aligned}
H H= & \sum_{k=-\infty}^{-1} 2^{k q \alpha(0)}\left\|\left[b, \mu_{\Omega}\right](f) \chi_{k}\right\|_{L^{p(\cdot)}}^{q} \\
\leq & \sum_{k=-\infty}^{-1} 2^{k q \alpha(0)}\left(\sum_{j=k}^{\infty}\left\|\left[b, \mu_{\Omega}\right]\left(f_{j}\right) \chi_{k}\right\|_{L^{p(\cdot)}}\right)^{q} \\
& +\sum_{k=-\infty}^{-1} 2^{k q \alpha(0)}\left(\sum_{j=-\infty}^{k-1}\left\|\left[b, \mu_{\Omega}\right]\left(f_{j}\right) \chi_{k}\right\|_{L^{p(\cdot)}}\right)^{q} \\
& :=H H_{1}+H H_{2} .
\end{aligned}
$$

When $0<q \leq 1$, we get 


$$
\begin{aligned}
& H H_{1}=\sum_{k=-\infty}^{-1} 2^{k q \alpha(0)}\left(\sum_{j=k}^{\infty}\left\|\left[b, \mu_{\Omega}\right]\left(f_{j}\right) \chi_{k}\right\|_{L^{p(\cdot)}}\right)^{q} \\
& \leq C\|b\|_{*}^{q}\|\Omega\|_{L^{s}\left(S^{n-1}\right)}^{q} \sum_{k=-\infty}^{-1} 2^{k q \alpha(0)}\left(\sum_{j=k}^{\infty}\left|\lambda_{j}\right|(k-j) 2^{\left[-j \alpha+(j-k)\left(n \delta_{2}-\gamma-\frac{n}{s}\right)\right]}\right)^{q} \\
& \leq C\|b\|_{*}^{q}\|\Omega\|_{L^{s}\left(S^{n-1}\right)}^{q} \sum_{k=-\infty}^{-1} 2^{k q \alpha(0)}\left(\sum_{j=k}^{\infty}\left|\lambda_{j}\right|^{q}(k-j)^{q} 2^{\left[-j \alpha(0)+(j-k)\left(n \delta_{2}-\gamma-\frac{n}{s}\right)\right]} q\right. \\
& \left.+\left.\sum_{j=k}^{\infty}\left|\lambda_{j}\right|\right|^{q}(k-j)^{q} 2^{\left[-j \alpha_{\infty}+(j-k)\left(n \delta_{2}-\gamma-\frac{n}{s}\right)\right]}\right] \\
& \leq C\|b\|_{*}^{q}\|\Omega\|_{L^{5}\left(s^{n-1}\right)}^{q} \sum_{k=-\infty}^{-1} \sum_{j=k}^{-1}\left|\lambda_{j}\right|^{q}(k-j)^{q} 2^{q(j-k)\left(n \delta_{2}-\gamma-\frac{n}{s}-\alpha(0)\right)} \\
& \left.\left.+C\|b\|_{*}^{q}\|\Omega\|_{L^{s}\left(S^{n-1}\right)}^{q} \sum_{k=-\infty}^{-1} 2^{k q \alpha(0)} \sum_{j=0}^{-\infty}\left|\lambda_{j}\right|^{q}(k-j) 2^{\left[-j \alpha_{\infty}+(j-k)\left(n \delta_{2}-\gamma-\frac{n}{s}\right)\right.}\right]\right]^{q} \\
& \leq C\|b\|_{*}^{q}\|\Omega\|_{L^{s}\left(s^{n-1}\right)}^{q} \sum_{k=-\infty}^{-1}\left|\lambda_{j}\right|^{q} \sum_{j=-\infty}^{j}(k-j)^{q} 2^{q(j-k)\left(n \delta_{2}-\gamma-\frac{n}{s}-\alpha(0)\right)} \\
& +C\|b\|_{*}^{q}\|\Omega\|_{L^{s}\left(S^{n-1}\right)}^{q} \sum_{k=-\infty}^{-1} 2^{k \alpha \alpha(0)} \sum_{j=0}^{\infty}\left|\lambda_{j}\right|^{q}(k-j) 2^{\left[-j \alpha_{\infty \infty}+(j-k)\left(n \delta_{2}-\gamma-\frac{n}{s}\right)\right] a} \\
& \leq C\|b\|_{*}^{q}\|\Omega\|_{L^{s}\left(S^{n-1}\right)}^{q} \sum_{k=-\infty}^{-1}\left|\lambda_{j}\right|^{q} \\
& +C\|b\|_{*}^{q}\|\Omega\|_{L^{s}\left(s^{n-1}\right)}^{q} \sum_{j=0}^{\infty}(k-j) 2^{\left[\left(\lambda-\alpha_{\infty}\right) j+(j-k)\left(n \delta_{2}-\gamma-\frac{n}{s}\right)\right] q} 2^{j \lambda q} \sum_{i=\infty}^{j}\left|\lambda_{i}\right| \sum_{k=-\infty}^{j} 2^{k q \alpha(0)} \\
& \leq C\|b\|_{*}^{q}\|\Omega\|_{L^{s}\left(s^{n-1}\right)}^{q} \Delta \\
& +C\|b\|_{*}^{q}\|\Omega\|_{L^{s}\left(S^{n-1}\right)}^{q} \Delta \sum_{j=0}^{\infty}(k-j) 2^{\left[\left(\lambda-\alpha_{\infty}\right) j+(j-k)\left(n \delta_{2}-\gamma-\frac{n}{s}\right)\right] q} \sum_{k=-\infty}^{j} 2^{k q \alpha(0)} \\
& \leq C\|b\|_{*}^{q}\|\Omega\|_{L^{s}\left(s^{n-1}\right)}^{q} \Delta .
\end{aligned}
$$

Now when $1<q<\infty$, let $1 / q+1 / q^{\prime}=1$ we have

$$
\begin{aligned}
& H H_{1}=\sum_{k=-\infty}^{-1} 2^{k q \alpha(0)}\left(\sum_{j=k}^{\infty}\left\|\left[b, \mu_{\Omega}\right]\left(f_{j}\right) \chi_{k}\right\|_{L^{p(\cdot)}}\right)^{q} \\
& \leq C\|b\|_{*}^{q}\|\Omega\|_{L^{s}\left(n^{n-1}\right)}^{q} \sum_{k=-\infty}^{-1} 2^{k q \alpha(0)}\left(\sum_{j=k}^{\infty}\left|\lambda_{j}\right|(k-j) 2^{\left[-j \alpha+(j-k)\left(n \delta_{2}-\gamma-\frac{n}{s}\right)\right]}\right)^{q} \\
& \left.\leq C\|b\|_{*}^{q}\|\Omega\|_{L^{s}\left(S^{n-1}\right)}^{q} \sum_{k=-\infty}^{-1}\left(\sum_{j=k}^{-1}\left|\lambda_{j}\right|(k-j) 2^{\left[(j-k)\left(n \delta_{2}-\gamma-\frac{n}{s}\right)-\alpha(0)\right.}\right]\right)^{q} \\
& \left.+C\|b\|_{*}^{q}\|\Omega\|_{L^{s}\left(S^{n-1}\right)}^{q} \sum_{k=-\infty}^{-1} 2^{k q \alpha(0)}\left(\sum_{j=0}^{\infty}\left|\lambda_{j}\right|(k-j) 2^{\left[-j \alpha_{\infty}+(j-k)\left(n \delta_{2}-\gamma-\frac{n}{s}\right)\right.}\right]\right)^{q} \\
& \left.\leq C\|b\|_{*}^{q}\|\Omega\|_{L^{s}\left(S^{n-1}\right)}^{q} \sum_{k=-\infty}^{-1}\left(\sum_{j=k}^{-1}\left|\lambda_{j}\right|^{q}(k-j)^{q} 2^{\left[(j-k)\left(n \delta_{2}-\gamma-\frac{n}{s}\right)-\alpha(0)\right]}\right]^{q / 2}\right)
\end{aligned}
$$




$$
\begin{aligned}
& \times\left(\sum_{j=k}^{-1}(k-j)^{q} 2^{\left[(j-k)\left(n \delta_{2}-\gamma-\frac{n}{s}\right)-\alpha(0)\right] q^{\prime} / 2}\right)^{q / q^{\prime}} \\
& \left.+C\|b\|_{*}^{q}\|\Omega\|_{L^{s}\left(s^{n-1}\right)}^{q} \sum_{k=-\infty}^{-1} 2^{k q \alpha(0)}\left(\sum_{j=0}^{\infty}\left|\lambda_{j}\right|^{q}(k-j)^{q} 2^{\left[-j \alpha_{\infty}+(j-k)\left(n \delta_{2}-\gamma-\frac{n}{s}\right)\right]}\right]^{q / 2}\right)^{q} \\
& \times\left(\sum_{j=0}^{\infty}(k-j)^{q} 2^{\left[-j \alpha_{\infty}+(j-k)\left(n \delta_{2}-\gamma-\frac{n}{s}\right)\right] q^{\prime} / 2}\right)^{q / q^{\prime}} \\
& \leq C\|b\|_{*}^{q}\|\Omega\|_{L^{s}\left(s^{n-1}\right)}^{q} \sum_{k=-\infty}^{-1}\left|\lambda_{j}\right|^{q} \sum_{k=-\infty}^{j}(k-j)^{q} 2^{\left[(j-k)\left(n \delta_{2}-\gamma-\frac{n}{s}\right)-\alpha(0)\right] q / 2} \\
& \left.\left.+C\|b\|_{*}^{q}\|\Omega\|_{L^{s}\left(S^{n-1}\right)}^{q} \sum_{k=-\infty}^{-1} 2^{k q \alpha(0)} \sum_{j=0}^{\infty}\left|\lambda_{j}\right|^{q}(k-j)^{q} 2^{\left[-j \alpha_{\infty}+(j-k)\left(n \delta_{2}-\gamma-\frac{n}{s}\right)\right.}\right]\right]^{/ 2} \\
& \leq C\|b\|_{*}^{q}\|\Omega\|_{L^{s}\left(s^{n-1}\right)}^{q} \sum_{k=-\infty}^{-1}\left|\lambda_{j}\right|^{q} \sum_{j=k}^{-1}(k-j)^{q} 2^{\left[(j-k)\left(n \delta_{2}-\gamma-\frac{n}{s}\right)-\alpha(0)\right] q / 2} \\
& \left.+C\|b\|_{*}^{q}\|\Omega\|_{L^{s}\left(S^{n-1}\right)}^{q} \sum_{j=0}^{\infty}\left|\lambda_{j}\right|^{q} 2^{k q\left(\alpha(0)-\left(n \delta_{2}-\gamma-\frac{n}{s}\right) / 2\right.}\right) \sum_{k=-\infty}^{-1}(k-j)^{q} 2^{\left(n \delta_{2}-\gamma-\frac{n}{s}-\alpha_{\infty}\right) j q / 2} \\
& \leq C\|b\|_{*}^{q}\|\Omega\|_{L^{s}\left(S^{n-1}\right)}^{q} \sum_{k=-\infty}^{-1}\left|\lambda_{j}\right|^{q} \\
& \left.+C\|b\|_{*}^{q}\|\Omega\|_{L^{s}\left(s^{n-1}\right)}^{q} \sum_{j=0}^{\infty}(k-j)^{q} 2^{\left[\left(\lambda-\alpha_{\infty} / 2\right) j+(j-k)\left(n \delta_{2}-\gamma-\frac{n}{s}\right) q / 2\right.}\right] 2^{-j q \lambda} \sum_{i=-\infty}^{j}\left|\lambda_{j}\right|^{q} \sum_{k=-\infty}^{-1} 2^{k q \alpha(0)} \\
& \leq C\|b\|_{*}^{q}\|\Omega\|_{L^{s}\left(s^{n-1}\right)}^{q} \Delta \\
& \left.+C\|b\|_{*}^{q}\|\Omega\|_{L^{s}\left(s^{n-1}\right)}^{q} \Delta \sum_{j=0}^{\infty}(k-j)^{q} 2^{\left[\left(\lambda-\alpha_{\infty} / 2\right) j+(j-k)\left(n \delta_{2}-\gamma-\frac{n}{s}\right) q / 2\right.}\right] \sum_{k=-\infty}^{-1} 2^{k q \alpha(0)} \\
& \leq C\|b\|_{*}^{q}\|\Omega\|_{L^{s}\left(s^{n-1}\right)}^{q} \Delta .
\end{aligned}
$$

For $H H_{2}$, when $0<q \leq 1$, by $n \delta_{2} \leq \alpha(0)<s+\delta+n \delta_{2}$ we get

$$
\begin{aligned}
H H_{2} & =\sum_{k=-\infty}^{-1} 2^{k q \alpha(0)}\left(\sum_{j=-\infty}^{k-1}\left\|\left[b, \mu_{\Omega}\right]\left(f_{j}\right) \chi_{k}\right\|_{L^{p(\cdot)}}\right)^{q} \\
& \leq C\|b\|_{*}^{q}\|\Omega\|_{L^{s}\left(s^{n-1}\right)}^{q} \sum_{k=-\infty}^{-1} 2^{k q \alpha(0)}\left(\sum_{j=-\infty}^{k-1}\left|\lambda_{j}\right|(k-j) 2^{\left[-j \alpha(0)+(j-k)\left(n \delta_{2}-\gamma-\frac{n}{s}\right)\right]}\right] \\
& \leq C\|b\|_{*}^{q}\|\Omega\|_{L^{s}\left(s^{n-1}\right)}^{q} \sum_{k=-\infty}^{-1} 2^{k q \alpha(0)}\left(\sum_{j=-\infty}^{k-1}\left|\lambda_{j}\right|^{q}(k-j)^{q} 2^{q\left[-j \alpha(0)+(j-k)\left(n \delta_{2}-\gamma-\frac{n}{s}\right)\right]}\right] \\
& \leq C\|b\|_{*}^{q}\|\Omega\|_{L^{s}\left(s^{n-1}\right)}^{q} \sum_{k=-\infty}^{-1}\left|\lambda_{j}\right|^{q} \sum_{k=j+1}^{-1}(k-j)^{q} 2^{q(j-k))\left(n \delta_{2}-\gamma-\frac{n}{s}-\alpha(0)\right)} \\
& \leq C\|b\|_{*}^{q}\|\Omega\|_{L^{s}\left(s^{n-1}\right)}^{q} \Delta .
\end{aligned}
$$

Now $1<q<\infty$, let $1 / q+1 / q^{\prime}=1$. Since $n \delta_{2} \leq \alpha(0)<s+\delta+n \delta_{2}$, by Hölder's inequality we have 


$$
\begin{aligned}
& H_{2}=\sum_{k=-\infty}^{-1} 2^{k q \alpha(0)}\left(\sum_{j=-\infty}^{k-1}\left\|\left[b, \mu_{\Omega}\right]\left(f_{j}\right) \chi_{k}\right\|_{L^{p(\cdot)}}\right)^{q} \\
& \leq C\|b\|_{*}^{q}\|\Omega\|_{L^{s}\left(s^{n-1}\right)}^{q} \sum_{k=-\infty}^{-1} 2^{k q \alpha(0)}\left(\sum_{j=-\infty}^{k-1}\left|\lambda_{j}\right|(k-j) 2^{-j \alpha(0)+(j-k)\left(n \delta_{2}-\gamma-\frac{n}{s}\right)}\right)^{q} \\
& \left.\leq C\|b\|_{*}^{q}\|\Omega\|_{L^{s}\left(s^{n-1}\right)}^{q} \sum_{k=-\infty}^{-1} 2^{k q \alpha(0)}\left(\sum_{j=-\infty}^{k-1}\left|\lambda_{j}\right|^{q}(k-j)^{q} 2^{\left[-j \alpha(0)+(j-k)\left(n \delta_{2}-\gamma-\frac{n}{s}\right)\right]}\right]\right) \\
& \times\left(\sum_{j=-\infty}^{k-1}(k-j) 2^{\left[-j \alpha(0)+(j-k)\left(n \delta_{2}-\gamma-\frac{n}{s}\right)\right] q^{\prime} / 2}\right)^{q / q^{\prime}} \\
& \left.\left.\leq C\|b\|_{*}^{q}\|\Omega\|_{L^{s}\left(s^{n-1}\right)}^{q} \sum_{k=-\infty}^{-1} 2^{k q \alpha(0)}\left(\sum_{j=-\infty}^{k-1}\left|\lambda_{j}\right|^{q}(k-j)^{q} 2^{\left[-j \alpha(0)+(j-k)\left(n \delta_{2}-\gamma-\frac{n}{s}\right)\right.}\right]\right]^{q / 2}\right) \\
& \leq C\|b\|_{*}^{q}\|\Omega\|_{L^{s}\left(s^{n-1}\right)}^{q} \sum_{k=-\infty}^{-1}|\lambda|^{q} \sum_{k=j+1}^{-1}(k-j)^{q} 2^{(j-k)\left(n \delta_{2}-\gamma-\frac{n}{s}-\alpha(0)\right) q / 2} \\
& \leq C\|b\|_{*}^{q}\|\Omega\|_{L^{s}\left(s^{n-1}\right)}^{q} \Delta .
\end{aligned}
$$

Thirdly, we estimate $H H H$, we need to show that there exists a positive constant $C$, such that $H H H \leq C \Delta$

$$
\begin{aligned}
H H H= & \sup _{L \leq 0, L \in \mathbb{Z}} 2^{-L \lambda q} \sum_{k=0}^{L} 2^{k q \alpha_{\infty}}\left\|\left[b, \mu_{\Omega}\right](f) \chi_{k}\right\|_{L^{p(\cdot)}}^{q} \\
\leq & \sup _{L \leq 0, L \in \mathbb{Z}} 2^{-L \lambda q} \sum_{k=0}^{L} 2^{k q \alpha_{\infty}}\left(\sum_{j=k}^{\infty}\left\|\left[b, \mu_{\Omega}\right]\left(f_{j}\right) \chi_{k}\right\|_{L^{p(\cdot)}}\right)^{q} \\
& +\sup _{L \leq 0, L \in \mathbb{Z}} 2^{-L \lambda q} \sum_{k=0}^{L} 2^{k q \alpha_{\infty}}\left(\sum_{j=-\infty}^{k-1}\left\|\left[b, \mu_{\Omega}\right]\left(f_{j}\right) \chi_{k}\right\|_{L^{p(\cdot)}}\right)^{q} \\
& :=H_{H}+H_{1}+H_{2} .
\end{aligned}
$$

When $0<q \leq 1$ by boundedness of $\left[b, \mu_{\Omega}\right]$ in $L^{p(\cdot)}$

$$
\begin{aligned}
& H H H_{1}=\sup _{L \leq 0, L \in \mathbb{Z}} 2^{-L \lambda q} \sum_{k=0}^{L} 2^{k q \alpha_{\infty}}\left(\sum_{j=k}^{\infty}\left\|\left[b, \mu_{\Omega}\right]\left(f_{j}\right) \chi_{k}\right\|_{L^{p(\cdot)}}\right)^{q} \\
& \leq \sup _{L \leq 0, L \in \mathbb{Z}} 2^{-L \lambda q} \sum_{k=0}^{L} 2^{k q \alpha_{\infty}} \sum_{j=k}^{\infty}\left\|\left[b, \mu_{\Omega}\right]\left(f_{j}\right) \chi_{k}\right\|_{L^{p(\cdot)}}^{q} \\
& \left.\leq C\|b\|_{*}^{q}\|\Omega\|_{L^{s}}^{q}\left(s^{n-1}\right) \sup _{L \leq 0, L \in \mathbb{Z}} 2^{-L \lambda q} \sum_{k=0}^{L} 2^{k q \alpha_{\infty}} \sum_{j=k}^{\infty}\left|\lambda_{j}\right|^{q}(k-j)^{q} 2^{q\left[-j \alpha_{j}+(j-k)\left(n \delta_{2}-\gamma-\frac{n}{s}\right)\right.}\right] \\
& \left.\leq C\|b\|_{*}^{q}\|\Omega\|_{L^{s}\left(s^{n-1}\right)}^{q} \sup _{L \leq 0, L \in \mathbb{Z}} 2^{-L \lambda q} \sum_{k=0}^{L} 2^{k q \alpha_{\infty}} \sum_{j=k}^{\infty}\left|\lambda_{j}\right|^{q}(k-j)^{q} 2^{q\left[-j \alpha_{\infty}+(j-k)\left(n \delta_{2}-\gamma-\frac{n}{s}\right)\right.}\right] \\
& \leq C\|b\|_{*}^{q}\|\Omega\|_{L^{s}\left(s^{n-1}\right)}^{q} \sup _{L \leq 0, L \in \mathbb{Z}} 2^{-L \lambda q} \sum_{j=0}^{L}\left|\lambda_{j}\right|^{q} \sum_{k=0}^{j}(k-j)^{q} 2^{q(j-k)\left(n \delta_{2}-\gamma-\frac{n}{s}-\alpha_{\infty}\right)} \\
& +C\|b\|_{*}^{q}\|\Omega\|_{L^{s}\left(s^{n-1}\right)}^{q} \sup _{L \leq 0, L \in \mathbb{Z}} 2^{-L \lambda q} \sum_{j=L}^{\infty}\left|\lambda_{j}\right|^{q} \sum_{k=0}^{L}(k-j)^{q} 2^{q(j-k)\left(n \delta_{2}-\gamma-\frac{n}{s}-\alpha_{\infty}\right)} \\
& \leq C\|b\|_{*}^{q}\|\Omega\|_{L^{s}\left(s^{n-1}\right)}^{q} \sup _{L \leq 0, L \in \mathbb{Z}} 2^{-L \lambda q} \sum_{j=0}^{L}\left|\lambda_{j}\right|^{q}
\end{aligned}
$$


$+C\|b\|_{*}^{q}\|\Omega\|_{L^{s}\left(S^{n-1}\right)}^{q} \sup _{L \leq 0, L \in \mathbb{Z}} \sum_{j=L}^{\infty} 2^{(j-L) q \lambda} 2^{-L \lambda q} \sum_{i=-\infty}^{j}\left|\lambda_{i}\right|^{q} \sum_{k=0}^{L}(k-j)^{q} 2^{q(j-k)\left(n \delta_{2}-\gamma-\frac{n}{s}-\alpha_{\infty}\right)}$

$\leq C\|b\|_{*}^{q}\|\Omega\|_{L^{s}\left(s^{n-1}\right)}^{q} \Delta+C\|b\|_{*}^{q}\|\Omega\|_{L^{s}\left(s^{n-1}\right)}^{q} \Delta \sum_{k=0}^{L}(k-j)^{q} 2^{q(j-k)\left(n \delta_{2}-\gamma-\frac{n}{s}-\alpha_{\infty}\right)}$.

Now when $0<q<\infty$, by boundedness of $\left[b, \mu_{\Omega}\right]$ in $L^{p(\cdot)}$, see ([20]) by Hölder's inequality we have

$$
\begin{aligned}
& H H H_{1}=\sup _{L \leq 0, L \in \mathbb{Z}} 2^{-L \lambda q} \sum_{k=0}^{L} 2^{k q \alpha_{\infty}}\left(\sum_{j=k}^{\infty}\left\|\left[b, \mu_{\Omega}\right]\left(f_{j}\right) \chi_{k}\right\|_{L^{p(\cdot)}}\right)^{q} \\
& \leq \sup _{L \leq 0, L \in \mathbb{Z}} 2^{-L \lambda q} \sum_{k=0}^{L} 2^{k q \alpha_{\infty}}\left(\sum_{j=k}^{\infty} \|\left.\left[b, \mu_{\Omega}\right]\left(f_{j}\right) \chi_{k}\right|_{L^{p(\cdot)}} ^{q / 2}\right) \times\left(\sum_{j=k}^{\infty}\left\|\left[b, \mu_{\Omega}\right]\left(f_{j}\right) \chi_{k}\right\|_{L^{p \cdot(\cdot)}}^{q^{\prime} / 2}\right)^{q / q^{\prime}} \\
& \leq \sup _{L \leq 0, L \in \mathbb{Z}} 2^{-L \lambda q} \sum_{k=0}^{L} 2^{k q \alpha_{\infty}}\left(\sum_{j=k}^{\infty}\left|\lambda_{j}\right|^{q}\left\|b_{j}\right\|_{L^{p(\cdot)}}^{q / 2}\right) \times\left(\left.\sum_{j=k}^{\infty}|| b_{j}\right|_{L^{p(\cdot)}} ^{q^{\prime / / 2}}\right)^{q / q^{\prime}} \\
& \leq \sup _{L \leq 0, L \in \mathbb{Z}} 2^{-L \lambda q} \sum_{k=0}^{L} 2^{k q \alpha_{\infty}}\left(\sum_{j=k}^{\infty}\left|\lambda_{j}\right|^{q}\left|B_{j}\right|^{-\alpha_{j} q /(2 n)}\right) \times\left(\sum_{j=k}^{\infty}\left|B_{j}\right|^{-\alpha_{j} q^{\prime \prime}(2 n)}\right)^{q / q^{\prime}} \\
& \leq \sup _{L \leq 0, L \in \mathbb{Z}} 2^{-L \lambda q} \sum_{k=0}^{L} 2^{\alpha_{\infty} k q / 2}\left(\sum_{j=k}^{\infty}\left|\lambda_{j}\right|^{q}\left|B_{j}\right|^{-\alpha_{j} q /(2 n)}\right) \\
& =\sup _{L \leq 0, L \in \mathbb{Z}} 2^{-L \lambda q} \sum_{j=0}^{L}\left|\lambda_{j}\right|^{q} \sum_{k=0}^{j} 2^{\alpha_{\infty}(k-j) q / 2}+\sup _{L \leq 0, L \in \mathbb{Z}} 2^{-L \lambda q} \sum_{j=L}^{\infty}\left|\lambda_{j}\right|^{q} \sum_{k=0}^{L} 2^{\alpha_{\infty}(k-j) q / 2} \\
& \leq \sup _{L \leq 0, L \in \mathbb{Z}} 2^{-L \lambda q} \sum_{k=L}^{\infty}\left|\lambda_{j}\right|^{q}+\sup _{L \leq 0, L \in \mathbb{Z}} \sum_{j=L}^{\infty} 2^{(j-L) q \lambda} 2^{-j \lambda q} \sum_{i=-\infty}^{j}\left|\lambda_{i}\right|^{q} \sum_{k=0}^{L} 2^{\alpha_{\infty}(k-j) q / 2} \\
& \leq \Delta+\Delta \sup _{L \leq 0, L \in \mathbb{Z}} \sum_{j=L}^{\infty} 2^{(j-L) q \lambda} 2^{\alpha_{\infty}(k-j) q / 2} \\
& \leq \Delta .
\end{aligned}
$$

We have $0<q \leq 1$, by $n \delta_{2} \leq \alpha(0), \alpha_{\infty}<s+\delta+n \delta_{2}$ we get

$H H H_{2}=\sup _{L \leq 0, L \in \mathbb{Z}} 2^{-L \lambda q} \sum_{k=0}^{L} 2^{k q \alpha_{\infty}}\left(\sum_{j=-\infty}^{k-1}\left\|\left[b, \mu_{\Omega}\right]\left(f_{j}\right) \chi_{k}\right\|_{L^{p(\cdot)}}\right)^{q}$

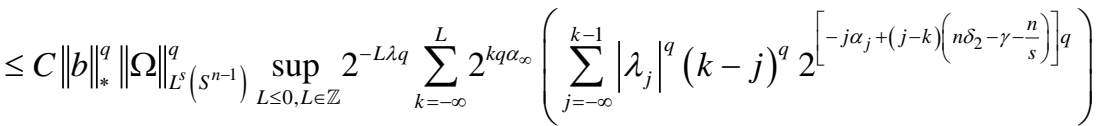

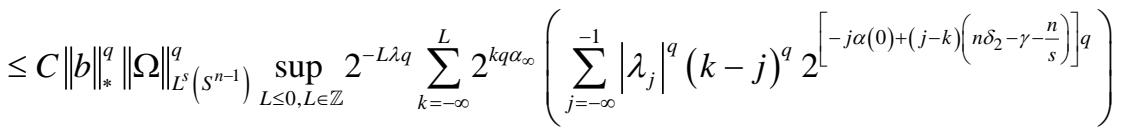
$+C\|b\|_{*}^{q}\|\Omega\|_{L^{s}\left(S^{n-1}\right)}^{q} \sup _{L \leq 0, L \in \mathbb{Z}} 2^{-L \lambda q} \sum_{k=0}^{L} 2^{k q \alpha_{\infty}}\left(\sum_{j=0}^{k-1}\left|\lambda_{j}\right|^{q}(k-j)^{q} 2^{\left[-j \alpha_{\infty}+(j-k)\left(n \delta_{2}-\gamma-\frac{n}{s}\right)\right] q}\right)$ $\leq C\|b\|_{*}^{q}\|\Omega\|_{L^{s}\left(s^{n-1}\right)}^{q} \sup _{L \leq 0, L \in \mathbb{Z}} 2^{-L \lambda q} \sum_{k=0}^{L} 2^{k q\left[\alpha_{\infty}+\gamma+n \delta_{2}\right]} \sum_{j=-\infty}^{-1}\left|\lambda_{j}\right|^{q}(k-j)^{q} 2^{\left[n \delta_{2}-\gamma-\frac{n}{s}-\alpha(0)\right] j q}$ $+C\|b\|_{*}^{q}\|\Omega\|_{L^{s}\left(S^{n-1}\right)}^{q} \sup _{L \leq 0, L \in \mathbb{Z}} 2^{-L \lambda q} \sum_{k=0}^{L}\left|\lambda_{j}\right|^{q} \sum_{j=0}^{k-1}(k-j)^{q} 2^{(j-k)\left(n \delta_{2}-\gamma-\frac{n}{s}-\alpha_{\infty}\right) q}$

$\leq C\|b\|_{*}^{q}\|\Omega\|_{L^{s}\left(s^{n-1}\right)}^{q} \sup _{L \leq 0, L \in \mathbb{Z}} 2^{-L \lambda q} \sum_{j=-\infty}^{-1}\left|\lambda_{j}\right|^{q}+C\|b\|_{*}^{q}\|\Omega\|_{L^{s}\left(S^{n-1}\right)}^{q} \sup _{L \leq 0, L \in \mathbb{Z}} 2^{-L \lambda q} \sum_{k=0}^{L-1}\left|\lambda_{j}\right|^{q}$ $\leq C\|b\|_{*}^{q}\|\Omega\|_{L^{s}\left(s^{n-1}\right)}^{q} \Delta$. 
Now when $1<q<\infty$, let $1 / q+1 / q^{\prime}=1$. Since $n \delta_{2} \leq \alpha(0), \alpha(\infty)<s+\delta+n \delta_{2}$, by Hölder's inequality, we have

$$
\begin{aligned}
& H H H_{2}=\sup _{L \leq 0, L \in \mathbb{Z}} 2^{-L \lambda q} \sum_{k=0}^{L} 2^{k q \alpha_{\infty}}\left(\sum_{j=-\infty}^{k-1}\left\|\left[b, \mu_{\Omega}\right]\left(f_{j}\right) \chi_{k}\right\|_{L^{p(\cdot)}}\right)^{q} \\
& \leq C\|b\|_{*}^{q}\|\Omega\|_{L^{s}\left(S^{n-1}\right)}^{q} \sum_{k=0}^{L} 2^{k q \alpha_{\infty}}\left(\sum_{j=-\infty}^{k-1}\left|\lambda_{j}\right|(k-j) 2^{-j \alpha_{j}+(j-k)\left(n \delta_{2}-\gamma-\frac{n}{s}\right)}\right)^{q} \\
& \leq C\|b\|_{*}^{q}\|\Omega\|_{L^{5}\left(s^{n-1}\right)}^{q} \sum_{k=0}^{L} 2^{k q \alpha_{\infty}}\left(\sum_{j=-\infty}^{-1}\left|\lambda_{j}\right|(k-j) 2^{-j \alpha(0)+(j-k)\left(n \delta_{2}-\gamma-\frac{n}{s}\right)}\right)^{q} \\
& +C\|b\|_{*}^{q}\|\Omega\|_{L^{5}\left(S^{n-1}\right)}^{q} \sum_{k=0}^{L} 2^{k \alpha \alpha_{\infty}}\left(\sum_{j=0}^{k-1}\left|\lambda_{j}\right|(k-j) 2^{-j \alpha_{\infty}+(j-k)\left(n \delta_{2}-\gamma-\frac{n}{s}\right)}\right)^{q} \\
& \left.\leq C\|b\|_{*}^{q}\|\Omega\|_{L^{s}\left(S^{n-1}\right)}^{q} \sum_{k=0}^{L} 2^{k q\left[\alpha_{\infty}-\left(n \delta_{2}-\gamma-\frac{n}{s}\right)\right.}\right]\left(\sum_{j=-\infty}^{-1}\left|\lambda_{j}\right|(k-j) 2^{j\left(n \delta_{2}-\gamma-\frac{n}{s}-\alpha(0)\right)}\right)^{q} \\
& +C\|b\|_{*}^{q}\|\Omega\|_{L^{s}\left(s^{n-1}\right)}^{q} \sum_{k=0}^{L}\left(\sum_{j=0}^{k-1}\left|\lambda_{j}\right|(k-j) 2^{(j-k)\left(n \delta_{2}-\gamma-\frac{n}{s}-\alpha_{\infty}\right)}\right)^{q} \\
& \leq C\|b\|_{*}^{q}\|\Omega\|_{L^{s}\left(S^{n-1}\right)}^{q}\left(\sum_{j=-\infty}^{-1}\left|\lambda_{j}\right|^{q}(k-j)^{q} 2^{j q / 2\left(n \delta_{2}-\gamma-\frac{n}{s}-\alpha(0)\right)}\right) \\
& \times\left(\sum_{j=-\infty}^{-1} 2^{q^{\prime} / 2 j\left(n \delta_{2}-\gamma-\frac{n}{s}-\alpha(0)\right)}\right)^{q / q^{\prime}} \\
& +C\|b\|_{*}^{q}\|\Omega\|_{L^{s}\left(S^{n-1}\right)}^{q} \sum_{k=0}^{L}\left(\sum_{j=0}^{k-1}\left|\lambda_{j}\right|^{q}(k-j)^{q} 2^{q / 2(j-k)\left(n \delta_{2}-\gamma-\frac{n}{s}-\alpha_{\infty}\right)}\right) \\
& \times\left(\sum_{j=0}^{k-1} 2^{q^{\prime} / 2(j-k)\left(n \delta_{2}-\gamma-\frac{n}{s}-\alpha_{\infty}\right)}\right)^{q / q^{\prime}} \\
& \leq C\|b\|_{*}^{q}\|\Omega\|_{L^{s}\left(S^{n-1}\right)}^{q} \sum_{j=-\infty}^{-1}\left|\lambda_{j}\right|^{q}(k-j)^{q} 2^{j q / 2\left(n \delta_{2}-\gamma-\frac{n}{s}-\alpha(0)\right)} \\
& +C\|b\|_{*}^{q}\|\Omega\|_{L^{s}\left(s^{n-1}\right)}^{q} \sum_{k=0}^{L} \sum_{j=0}^{k-1}\left|\lambda_{j}\right|^{q}(k-j)^{q} 2^{q / 2(j-k)\left(n \delta_{2}-\gamma-\frac{n}{s}-\alpha_{\infty}\right)} \\
& \leq C\|b\|_{*}^{q}\|\Omega\|_{L^{5}\left(S^{n-1}\right)}^{q} \sum_{j=-\infty}^{-1}\left|\lambda_{j}\right|^{q} \\
& +C\|b\|_{*}^{q}\|\Omega\|_{L^{s}\left(S^{n-1}\right)}^{q} \sum_{k=0}^{L-1}\left|\lambda_{j}\right|^{q} \sum_{k=j+1}^{L}(k-j)^{q} 2^{q / 2(j-k)\left(n \delta_{2}-\gamma-\frac{n}{s}-\alpha_{\infty}\right)} \\
& \leq C\|b\|_{*}^{q}\|\Omega\|_{L^{s}\left(S^{n-1}\right)}^{q} \sum_{j=-\infty}^{-1}\left|\lambda_{j}\right|^{q}+C\|b\|_{*}^{q}\|\Omega\|_{L^{s}\left(S^{n-1}\right)}^{q} \sum_{k=0}^{L-1}\left|\lambda_{j}\right|^{q} \\
& \leq C\|b\|_{*}^{q}\|\Omega\|_{L^{s}\left(s^{n-1}\right)}^{q} \Delta .
\end{aligned}
$$

Joint the estimates for $\mathrm{H}, \mathrm{HH}$ and $\mathrm{HHH}$, we obtain

$$
\left\|\left[b, \mu_{\Omega}\right](f)\right\|_{M K_{p(\cdot), \lambda}^{\alpha(P,) q}\left(\mathbb{R}^{n}\right)}^{q} \leq C\|b\|_{*}^{q}\|\Omega\|_{L^{5}\left(S^{n-1}\right)}^{p}\|f\|_{H M K_{p(), \lambda}^{\alpha(\cdot), q}} \cdot
$$


Then we complete the proof of Theorem 4.1.

\section{Conclusion}

The study concluded that we can proof of boundedness for commutator of Marcinkiewicz integrals on Herz-Morrey-Hrdy spaces with variable exponent, which we use The main tools are properties of variable exponent in theorem 3.1 when $b \in \operatorname{Lip}_{\gamma}\left(\mathbb{R}^{n}\right)$, in theorem 4.1 when $b \in B M O\left(\mathbb{R}^{n}\right)$. We can obtain a solution for proof that commutator of Marcinkiewicz integrals are boundedness.

\section{Acknowledgements}

The authors cordially thank the reviewers for their valuable suggestions and useful comments which have led to the improvement of this paper.

\section{Conflicts of Interest}

The authors declare no conflicts of interest regarding the publication of this paper.

\section{References}

[1] Marcinkiewicz, J. (1938) Sur quelques integrales du type de Dini. Annales de la Societe Polonaise de Mathematique, 17, 42-50.

[2] Stein, E.M. (1958) On the Function of Little Wood-Paley, Lusin and Marcinkiewicz. Transactions of the American Mathematical Society, 88, 430-466. https://doi.org/10.1090/S0002-9947-1958-0112932-2

[3] Ding, Y., Fan, D. and Pan, Y. (1999) Weighted Boundedness for a Class of Rough Marcinkiewicz Integrals. Indiana University Mathematics Journal, 48, 1037-1056. https://doi.org/10.1512/iumj.1999.48.1696

[4] Torchinsky, A. and Wang, S. (1990) A Note on the Marcinkiewicz Integral. Colloquium Mathematicum, 60, 235-243. https://doi.org/10.4064/cm-60-61-1-235-243

[5] Xu, J. and Yang, X. (2016) The Molecular Characterization of Herz-Morrey-Haredy Space. Journal of Mathematical Inequalities, 10, 977-1008. https://doi.org/10.7153/jmi-10-79

[6] Xu, J. and Yang, X. (2015) Herz-Morrey-Hardy Spaces with Variable Exponents and Their Applications. Journal of Function Spaces, 2015, Article ID: 160635, 19 p. https://doi.org/10.1155/2015/160635

[7] Omer, A. and Tao S. (2019) Boundedness of Commutators on Herz-Morry-Hardy Spaces with Variable Exponents. Open Journal of Mathematical Science, 3, 94-108. https://doi.org/10.30538/oms2019.0052

[8] Yang, Y. and Tao, S. (2018) Singular Integrals with Variable Kernel and Fractional Differentiation in Homogeneous Morrey-Herz-Type Hardy Spaces with Variable Exponents. Open Mathematics, 16, 326-345. https://doi.org/10.1515/math-2018-0036

[9] Afiff, A., Omer, A. and Tao, S.P. (2018) Boundedness of Fractional Marcinkiewicz Integral with Variable Kernel on Variable Morrey-Herz Spaces. Open Journal of Mathematical Science, 2, 93-114. https://doi.org/10.30538/oms2018.0020

[10] Nantomah, K. (2017) Generalized Hölder's and Minkowski’s Inequalities for Jackson's q-Integral and Some Applications to the Incomplete q-Gamma Function. $A b$ - 
stract and Applied Analysis, 2017, Article ID: 9796873. https://doi.org/10.1155/2017/9796873

[11] Wu, J. and Wen, J. (2017) Boundedness for Higher Order Commutators of Fractional Integrals on Variable Exponent Herz-Morrey Spaces. Mediterranean Journal of Mathematics, 14, Article No. 198. https://doi.org/10.1007/s00009-017-1002-y

[12] Tan, J. and Liu, Z. (2015) Some Boundedness of Homogeneous Fractional Integrals on Variable Exponent Function Spaces. Acta Mathematica Sinica (Chinese Series), 58, 309-320.

[13] Izuki, M. (2010) Boundedness of Commutators on Herz Spaces with Variable Exponent. Rendiconti del Circolo Matematico di Palermo, 59, 199-213. https://doi.org/10.1007/s12215-010-0015-1

[14] Wang, H.B. and Liao, F. (2020) Boundedness of Singular Integral Operators on Herz-Morrey Spaces with Variable Exponent. Chinese Annals of Mathematics, Series $B, 41$, 99-116. https://doi.org/10.1007/s11401-019-0188-7

[15] Wang, H.B. (2020) The Continuity for Commutator of Maricnkiewicz Integrals on Herz-Type Space with Variable Exponent. Journal of University of Chinese Academy of Sciences, 37, 155-161.

[16] Wang, L.W. (2014) Marcinkiewicz Integral Operators and Commutators on Herz Spaces with Variable Exponents. Journal of Function Spaces, 2014, Article ID: 430365. https://doi.org/10.1155/2014/430365

[17] Xuan, Z.-H. and Shu, L.-S. (2013) Boundedness of Higher order Commutators on Herz-Morrey Spaces with Variable Exponent. Journal of Nanjing University Mathematical Biquarterly, 30, 188-196.

[18] Yong, D., Shan, Z. and Qing, X. (2002) Maricnkiewicz Integral on Hardy Spaces. Integral Equations and Operator Theory, 42, 174-182.

https://doi.org/10.1007/BF01275514

[19] Wang, H. (2017) Commutators of Singular Integuler Operator on Herz-Type Hardy Spaces with Variable Exponents. Journal of the Korean Mathematical Society, 54, 713-732. https://doi.org/10.4134/JKMS.j150771

[20] Tao, Sh.P. and Li, L.L. (2016) Boundednesss of Marcikiewicz Integrals and Commutators on Morrey Spaces with Varible Exponent. Chinese Journal of Contemporary Mathematics, 37, 53-68. 